\title{
An Enantioselective Chiral Bronsted Acid Catalyzed Imino-Azaenamine Reaction
}

\author{
Magnus Rueping, Erli Sugiono, Thomas Theissmann, Alexander Künkel, \\ Angela Köckritz, Anahit Pews-Davtyan, Navid Nemati, Matthias Beller
}

\section{Supporting Information}

General: All reactions were performed under argon atmosphere. Unless otherwise noted, all materials were obtained from commercial suppliers and were used without further purification. Solvents for extraction and chromatography were technical grade and distilled prior to use. Solvents used in reaction were reagent grade and distilled from the indicated drying agents: $\mathrm{CH}_{2} \mathrm{Cl}_{2}\left(\mathrm{P}_{2} \mathrm{O}_{5}\right)$, toluene $(\mathrm{Na}), \mathrm{CHCl}_{3}\left(\mathrm{CaH}_{2}\right)$. For thin-layer chromatography (TLC), silica gel coated aluminum plates (Merck, silica gel $60 \mathrm{~F}_{254}$ ) were used and chromatograms were visualised by irradiation with UV light at $254 \mathrm{~nm}$. Column chromatography was performed using silica gel Merck 60 (particle size $0.063-0.20 \mathrm{~mm}$ ). Solvents mixtures are understood as volume / volume.

${ }^{1} \mathrm{H}-\mathrm{NMR}$ and ${ }^{13} \mathrm{C}-\mathrm{NMR}$ were recorded on a Bruker AM 250 spectrometer in $\mathrm{CDCl}_{3}$. Data are reported in the following order: chemical shift $(\delta)$ in ppm; multiplicities are indicated (bs (broadened singlet), s (singlet), d (doublet), t (triplet), m (multiplet)); coupling constants $(J)$ are in Hertz $(\mathrm{Hz})$. High resolution mass spectra (HRMS-ESI) were conducted on Micromass/ Waters Qtof Ultima 3 instrument. IR spectra were recorded on a Jasco FT/IR-420 spectrometer and are reported in terms of frequency of absorption (cm-1). The enantiomeric excesses were determined by HPLC analysis using a chiral stationary phase column (column, Daicel Co. CHIRALCEL OD-H and AD-H; eluent: $n$-hexane / 2-propanol). The chiral HPLC method were calibrated with the corresponding racemic mixtures. Optical rotations were measured on a Perkin Elmer 241 polarimeter.

General Procedure: In a typical experiment the imine 2 ( $0.12 \mathrm{mmol}, 1.2$ equiv), catalyst (5 mol \%), azaenamine $1\left(0.10 \mathrm{mmol}, 1\right.$ equiv) and $\mathrm{CHCl}_{3}(0.7 \mathrm{~mL})$ were added to a screwcapped test tube at $\mathrm{rt}$. The resulting solution was cooled down to $0{ }^{\circ} \mathrm{C}$ and allowed to stir at the temperature for $16 \mathrm{~h}$. The solvent was evaporated in vacuo, and the residue was purified by column chromatography on silica gel (hexane/ethyl acetate, $5 / 1$ ) to give the hydrazone 3 . 


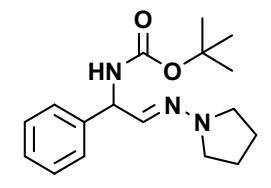

Hydrazone 3a: colorless solid, mp: $76{ }^{\circ} \mathrm{C},{ }^{1} \mathrm{H}-\mathrm{NMR}\left(\mathrm{CDCl}_{3}\right): \delta=1.45,1.49$ (2 bs, 9H, boc rotamers), $1.85-1.96(\mathrm{~m}, 4 \mathrm{H}), 3.10-3.25(\mathrm{~m}, 4 \mathrm{H}), 5.30$ (bs, $1 \mathrm{H}), 6.09(\mathrm{bs}, 1 \mathrm{H}), 6.52(\mathrm{bs}, 1 \mathrm{H}), 7.22-7.58(\mathrm{~m}, 5 \mathrm{H}) ;{ }^{13} \mathrm{C}-\mathrm{NMR}\left(\mathrm{CDCl}_{3}\right)$ : $\delta=155.1,132.9,128.6,128.0,127.3,127.0,125.8,79.3$; HRMS-ESI: calculated for $\mathrm{C}_{17} \mathrm{H}_{25} \mathrm{~N}_{3} \mathrm{O}_{2} \mathrm{Na}\left([\mathrm{M}+\mathrm{Na}]^{+}\right): 326.1844$, found: 326.1852; IR (neat): 3342, 2929, 1712, 1366, $700 \mathrm{~cm}^{-1}$; HPLC conditions: OD-H column, hexane $/ 2$-propanol $=98 / 2$, flow rate $=0.6 \mathrm{~mL}$ $\min ^{-1}$, minor enantiomer: $t_{R}=14,71$ min; major enantiomer: $t_{R}=16,90 \mathrm{~min} ;[\alpha]_{25}^{\mathrm{D}}=+33.3$ $\left(\mathrm{c}=1, \mathrm{CHCl}_{3}\right)$

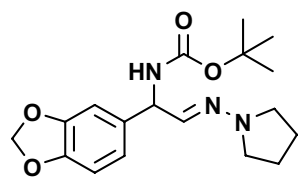

Hydrazone 3b: clear colorless oil, ${ }^{1} \mathrm{H}-\mathrm{NMR}\left(\mathrm{CDCl}_{3}\right): \delta=1.33(\mathrm{bs}, 9 \mathrm{H})$, $1.75-1.85(\mathrm{~m}, 4 \mathrm{H}), 3.06(\mathrm{t}, J=6.5 \mathrm{~Hz}, 4 \mathrm{H}), 5.08(\mathrm{bs}, 1 \mathrm{H}), 5.80-5.90$ $(\mathrm{m}, 2 \mathrm{H}), 5.97(\mathrm{~s}, 1 \mathrm{H}), 6.35(\mathrm{~s}, 1 \mathrm{H}), 6.61-6.78(\mathrm{~m}, 3 \mathrm{H}) ;{ }^{13} \mathrm{C}-\mathrm{NMR}$ $\left(\mathrm{CDCl}_{3}\right): \delta=155.0,147.8,146.8,135.5,132.8,120.4,108.2,107.4,100.9,89.2,79.3,51.3$, 28.4, 23.2; HRMS-ESI: calculated for $\mathrm{C}_{18} \mathrm{H}_{25} \mathrm{~N}_{3} \mathrm{O}_{4} \mathrm{Na}\left([\mathrm{M}+\mathrm{Na}]^{+}\right)$: 370.1743 , found: 370.1728 ; IR (neat): 3413, 2975, 1717, 1486, 1246, 1168, $1039 \mathrm{~cm}^{-1}$; HPLC conditions: AD-H column, hexane $/ 2$-propanol $=90 / 10$, flow rate $=0.6 \mathrm{~mL} \mathrm{~min}^{-1}$, minor enantiomer: $\mathrm{t}_{\mathrm{R}}=22,87 \mathrm{~min}$; major enantiomer: $\mathrm{t}_{\mathrm{R}}=24,94 \mathrm{~min} ;[\alpha]_{25}^{\mathrm{D}}=+54.9 \quad\left(\mathrm{c}=1, \mathrm{CHCl}_{3}\right)$

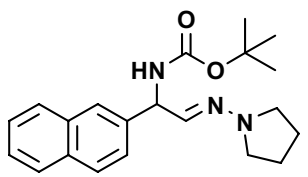

Hydrazone 3c: clear yellowish oil, ${ }^{1} \mathrm{H}-\mathrm{NMR}\left(\mathrm{CDCl}_{3}\right): \delta=1.50$ (bs, 9H), $1.76-2.04(\mathrm{~m}, 4 \mathrm{H}), 3.06-3.32(\mathrm{~m}, 4 \mathrm{H}), 6.10$ (bs, 2H), 6.67 (bs, 1H), $7.40-7.69(\mathrm{~m}, 4 \mathrm{H}), 7.82(\mathrm{~d}, J=7.5 \mathrm{~Hz}, 1 \mathrm{H}), 7.92(\mathrm{dd},, J=2.0 \mathrm{~Hz}, 7.5$ $\mathrm{Hz}, 1 \mathrm{H}), 8.29(\mathrm{~d}, J=8.3 \mathrm{~Hz}, 1 \mathrm{H}) ;{ }^{13} \mathrm{C}-\mathrm{NMR}\left(\mathrm{CDCl}_{3}\right): \delta=155.2,134.1,131.0,128.8,128.0$, 126.1, 125.5, 124.3, 123.4, 79.4, 51.3, 28.4, 23.2; HRMS-ESI: calculated for $\mathrm{C}_{21} \mathrm{H}_{27} \mathrm{~N}_{3} \mathrm{O}_{2} \mathrm{Na}$ $\left([\mathrm{M}+\mathrm{Na}]^{+}\right): 376.2001$, found: 376.2002; IR (neat): 3416, 2975, 1711, 1486, 1168, $779 \mathrm{~cm}^{-1}$; HPLC conditions: AD-H column, hexane $/ 2$-propanol $=95 / 5$, flow rate $=0.6 \mathrm{~mL} \mathrm{~min}{ }^{-1}$, major enantiomer: $t_{R}=27,83 \mathrm{~min}$; minor enantiomer: $t_{R}=32,93 \mathrm{~min} ;[\alpha]_{25}^{\mathrm{D}}=+38.7 \quad(\mathrm{c}=1$, $\left.\mathrm{CHCl}_{3}\right)$

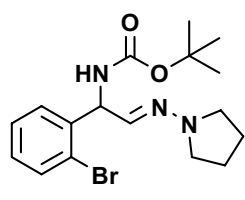

Hydrazone 3d: white needles, mp: $115{ }^{\circ} \mathrm{C},{ }^{1} \mathrm{H}-\mathrm{NMR}\left(\mathrm{CDCl}_{3}\right): \delta=1.17$, $1.36(2 \mathrm{bs}, 9 \mathrm{H}$, boc rotamers), $1.72-1.88(\mathrm{~m}, 4 \mathrm{H}), 3.05(\mathrm{t}, J=6.5 \mathrm{~Hz}, 4 \mathrm{H})$, $5.64(\mathrm{bs}, 1 \mathrm{H}), 6.17(\mathrm{bs}, 1 \mathrm{H}), 6.50(\mathrm{bs}, 1 \mathrm{H}), 6.95-7.10(\mathrm{~m}, 1 \mathrm{H}), 7.12-7.34$ (m, 2H), $7.45(\mathrm{dd}, J=1.3,6.8 \mathrm{~Hz}, 1 \mathrm{H}) ;{ }^{13} \mathrm{C}-\mathrm{NMR}\left(\mathrm{CDCl}_{3}\right): \delta=154.8,132.9,130.7,128.6$, 127.8, 122.7, 79.5, 51.2, 28.2, 23.2; HRMS-ESI: calculated for $\mathrm{C}_{17} \mathrm{H}_{24} \mathrm{~N}_{3} \mathrm{O}_{2} \mathrm{NaBr}\left([\mathrm{M}+\mathrm{Na}]^{+}\right)$ $($ Br-pattern) $=404.0950$, found: 404.0943; IR (neat): 3413, 2933, 1717, 1483, 1467, 1390, 
1249, 1168, 1022, 861, $725 \mathrm{~cm}^{-1}$; HPLC conditions: OD-H column, hexane / 2-propanol = 99 $/ 1$, flow rate $=0.6 \mathrm{~mL} \mathrm{~min}{ }^{-1}$, minor enantiomer: $t_{R}=20,47$ min; major enantiomer: $t_{R}=22,90$ $\min ;[\alpha]_{25}^{\mathrm{D}}=+53.5 \quad\left(\mathrm{c}=1, \mathrm{CHCl}_{3}\right)$

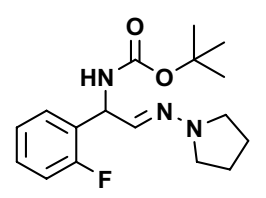

Hydrazone 3e: clear colorless oil, ${ }^{1} \mathrm{H}-\mathrm{NMR}\left(\mathrm{CDCl}_{3}\right): \delta=1.35,1.41$ (2bs, 9H, boc rotamers), $1.71-1.87(\mathrm{~m}, 4 \mathrm{H}), 3.06(\mathrm{t}, J=6.5 \mathrm{~Hz}, 4 \mathrm{H}), 5.49$ (bs, $1 \mathrm{H}), 5.99$ (bs, 1H), 6.45 (bs, 1H), $6.95-7.06$ (m, 2H), 7.08 - 7.28 (m, 2H); ${ }^{13} \mathrm{C}-\mathrm{NMR}\left(\mathrm{CDCl}_{3}\right): \delta=154.9,138.1,128.8,128.6,128.0,124.4,124.3,79.5,51.2,28.3,23.2$; HRMS-ESI: calculated for $\mathrm{C}_{17} \mathrm{H}_{24} \mathrm{~N}_{3} \mathrm{O}_{2} \mathrm{NaF}\left([\mathrm{M}+\mathrm{Na}]^{+}\right)$: 344.1750, found: 344.1756; IR (neat): 3421, 2976, 1699, 1460, 1366, 1171, $758 \mathrm{~cm}^{-1}$; HPLC conditions: AD-H column, hexane $/ 2$-propanol $=95 / 5$, flow rate $=0.6 \mathrm{~mL} \mathrm{~min}^{-1}$, minor enantiomer: $\mathrm{t}_{\mathrm{R}}=15,56 \mathrm{~min}$; major enantiomer: $\mathrm{t}_{\mathrm{R}}=18,28 \mathrm{~min} ;[\alpha]_{25}^{\mathrm{D}}=+31.2 \quad\left(\mathrm{c}=1, \mathrm{CHCl}_{3}\right)$

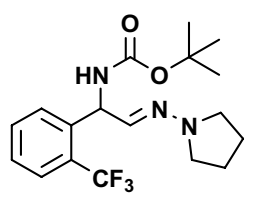

Hydrazone 3f: clear colorless oil, ${ }^{1} \mathrm{H}-\mathrm{NMR}\left(\mathrm{CDCl}_{3}\right): \delta=1.11,1.34$ (2 bs, 9H, boc rotamers), $1.70-1.88(\mathrm{~m}, 4 \mathrm{H}), 3.04(\mathrm{t}, J=6.8 \mathrm{~Hz}, 4 \mathrm{H}), 5.60$ (bs, $1 \mathrm{H}), 6.32(\mathrm{~d}, J=4.8 \mathrm{~Hz}, 2 \mathrm{H}), 7.10-7.34(\mathrm{~m}, 1 \mathrm{H}), 7.38-7.66(\mathrm{~m}, 3 \mathrm{H}) ;{ }^{13} \mathrm{C}-$ $\operatorname{NMR}\left(\mathrm{CDCl}_{3}\right): \delta=154.6,132.3,127.6,126.9,126.6,122.3,79.3,51.2,28.3,23.1$; HRMSESI: calculated for $\mathrm{C}_{18} \mathrm{H}_{24} \mathrm{~N}_{3} \mathrm{O}_{3} \mathrm{NaF}_{3}\left([\mathrm{M}+\mathrm{Na}]^{+}\right)$: 410,1667, found: 410,1658; IR (neat): 3317, 2972, 1718, 1483, 1313, 1159, $1035 \mathrm{~cm}^{-1}$; HPLC conditions: OD-H column, hexane / 2propanol $=99 / 1$, flow rate $=0.6 \mathrm{~mL} \mathrm{~min}^{-1}$, major enantiomer: $t_{\mathrm{R}}=15,73 \mathrm{~min}$; minor enantiomer: $\mathrm{t}_{\mathrm{R}}=18,18 \mathrm{~min} ;[\alpha]_{25}^{\mathrm{D}}=+37.2\left(\mathrm{c}=1, \mathrm{CHCl}_{3}\right)$

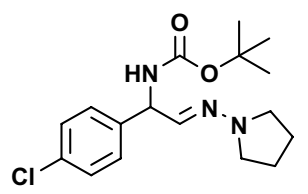

Hydrazone 3g: pale yellowish solid, mp: $105{ }^{\circ} \mathrm{C},{ }^{1} \mathrm{H}-\mathrm{NMR}\left(\mathrm{CDCl}_{3}\right): \delta=$ 1.32 (bs, 9H), $1.72-1.88$ (m, 4H), 3.06 (t, $J=6.5 \mathrm{~Hz}, 4 \mathrm{H}), 5.13$ (bs, 1H), $6.02(\mathrm{bs}, 1 \mathrm{H}), 6.33(\mathrm{bs}, 1 \mathrm{H}), 7.14-7.28(\mathrm{~m}, 4 \mathrm{H}) ;{ }^{13} \mathrm{C}-\mathrm{NMR}\left(\mathrm{CDCl}_{3}\right): \delta=$ $155.0,133.0,128.7,128.4,128.2,79.5,51.2,28.3,23.2$; HRMS-ESI: calculated for $\mathrm{C}_{17} \mathrm{H}_{24} \mathrm{~N}_{3} \mathrm{O} 2 \mathrm{NaCl}\left([\mathrm{M}+\mathrm{Na}]^{+}\right): 360.1455$, found: 360.1468; IR (neat): 3417, 2975, 1711, 1487 , 1166, $829 \mathrm{~cm}^{-1}$; HPLC conditions: AD-H column, hexane $/ 2$-propanol $=95 / 5$, flow rate $=$ $0.6 \mathrm{~mL} \min ^{-1}$, major enantiomer: $t_{R}=20,83 \mathrm{~min}$; minor enantiomer: $t_{R}=24,03 \mathrm{~min} ;[\alpha]^{\mathrm{D}}{ }_{25}=$ $+54.6\left(\mathrm{c}=1, \mathrm{CHCl}_{3}\right)$

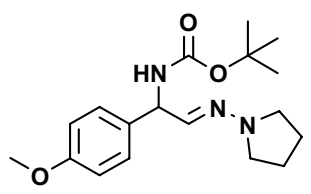

Hydrazone 3h: clear colorless oil, ${ }^{1} \mathrm{H}-\mathrm{NMR}\left(\mathrm{CDCl}_{3}\right): \delta=1.33(\mathrm{bs}, 9 \mathrm{H})$, $1.70-1.86(\mathrm{~m}, 4 \mathrm{H}), 3.06(\mathrm{t}, J=6.5 \mathrm{~Hz}, 4 \mathrm{H}), 3.72(\mathrm{~s}, 3 \mathrm{H}), 5.13(\mathrm{bs}, 1 \mathrm{H})$, 
$5.93(\mathrm{bs}, 1 \mathrm{H}), 6.39$ (bs, $1 \mathrm{H}), 6.79(\mathrm{~d}, J=8.8 \mathrm{~Hz}, 2 \mathrm{H}), 7.17$ (d, $J=8.8 \mathrm{~Hz}, 2 \mathrm{H}) ;{ }^{13} \mathrm{C}-\mathrm{NMR}$ $\left(\mathrm{CDCl}_{3}\right): \delta=158.8,155.1,128.3,113.9,79.2,55.2,51.3,28.4,23.1$; HRMS-ESI: calculated for $\mathrm{C}_{18} \mathrm{H}_{27} \mathrm{~N}_{3} \mathrm{O}_{3} \mathrm{Na}\left([\mathrm{M}+\mathrm{Na}]^{+}\right)$: 356.1950, found: 356.1960; IR (neat): 3415, 2974, 1713, 1511, 1248, 1169, $833 \mathrm{~cm}^{-1}$; HPLC conditions: AD-H column, hexane / 2-propanol = 95 / 5, flow

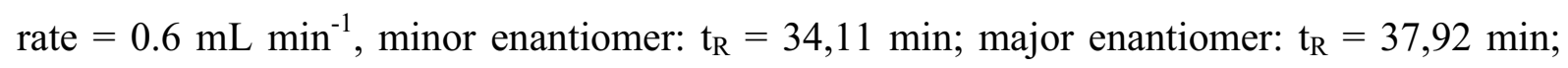
$[\alpha]_{25}^{\mathrm{D}}=+55,7 \quad\left(\mathrm{c}=1, \mathrm{CHCl}_{3}\right)$ 


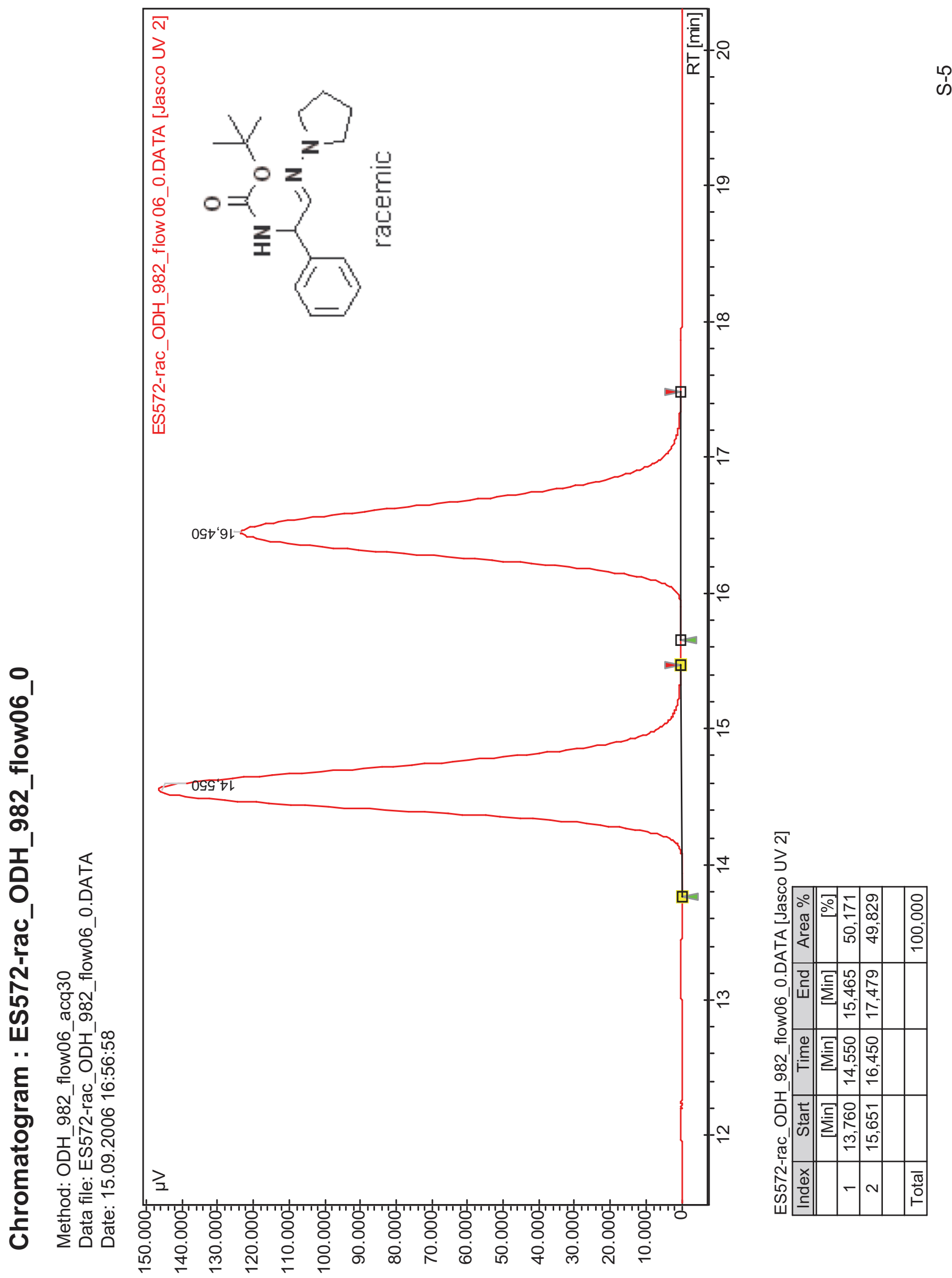




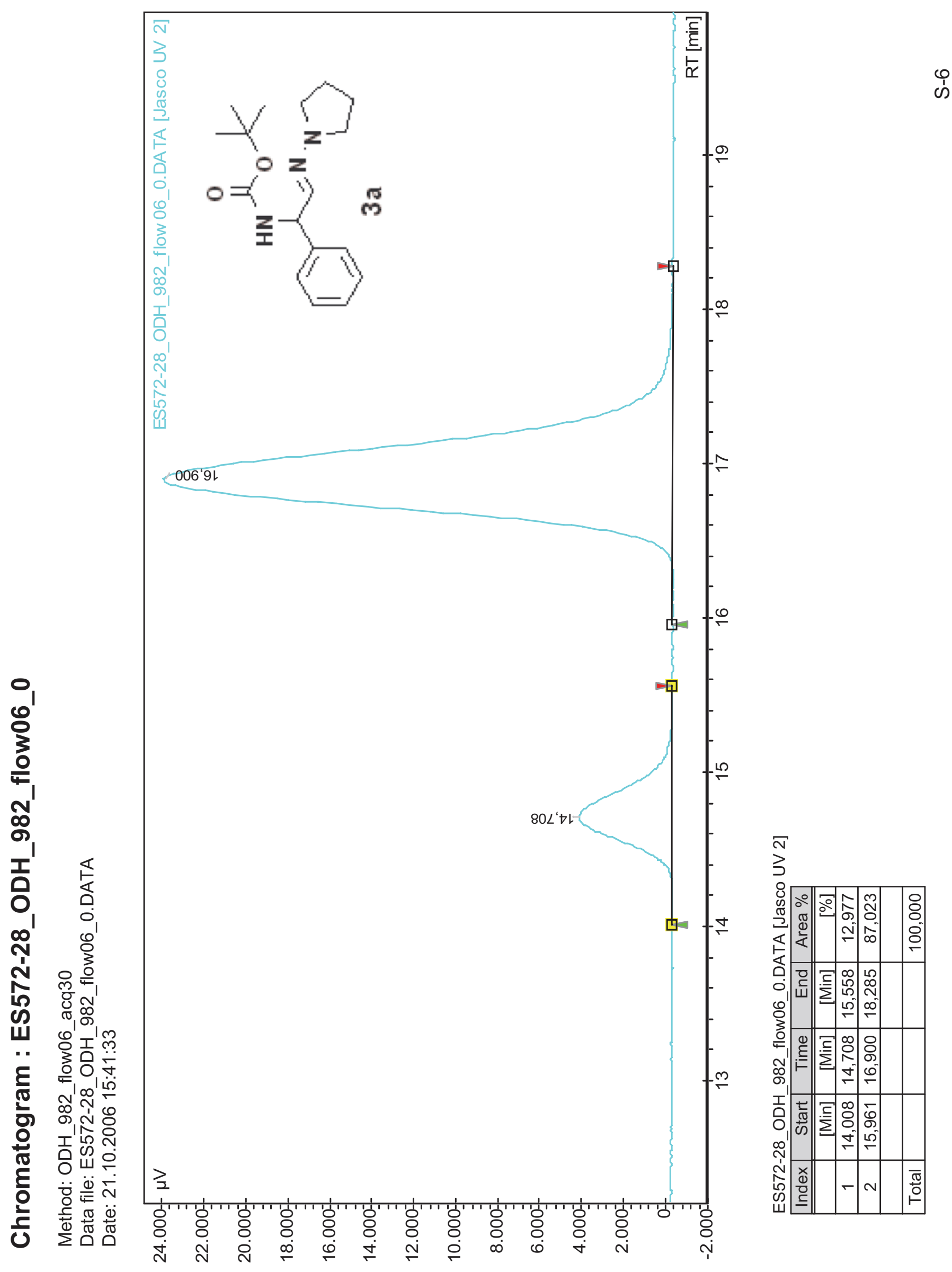




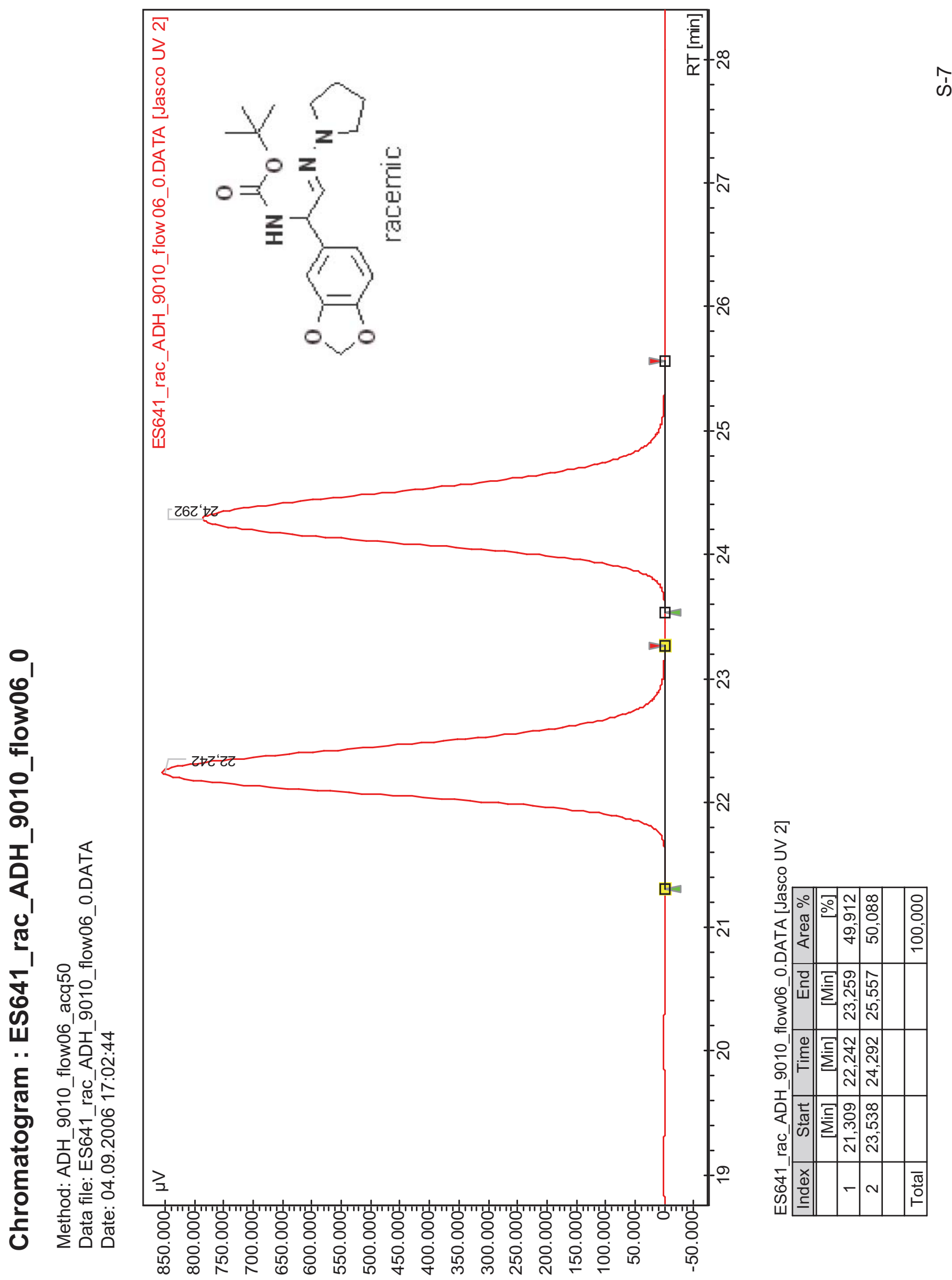




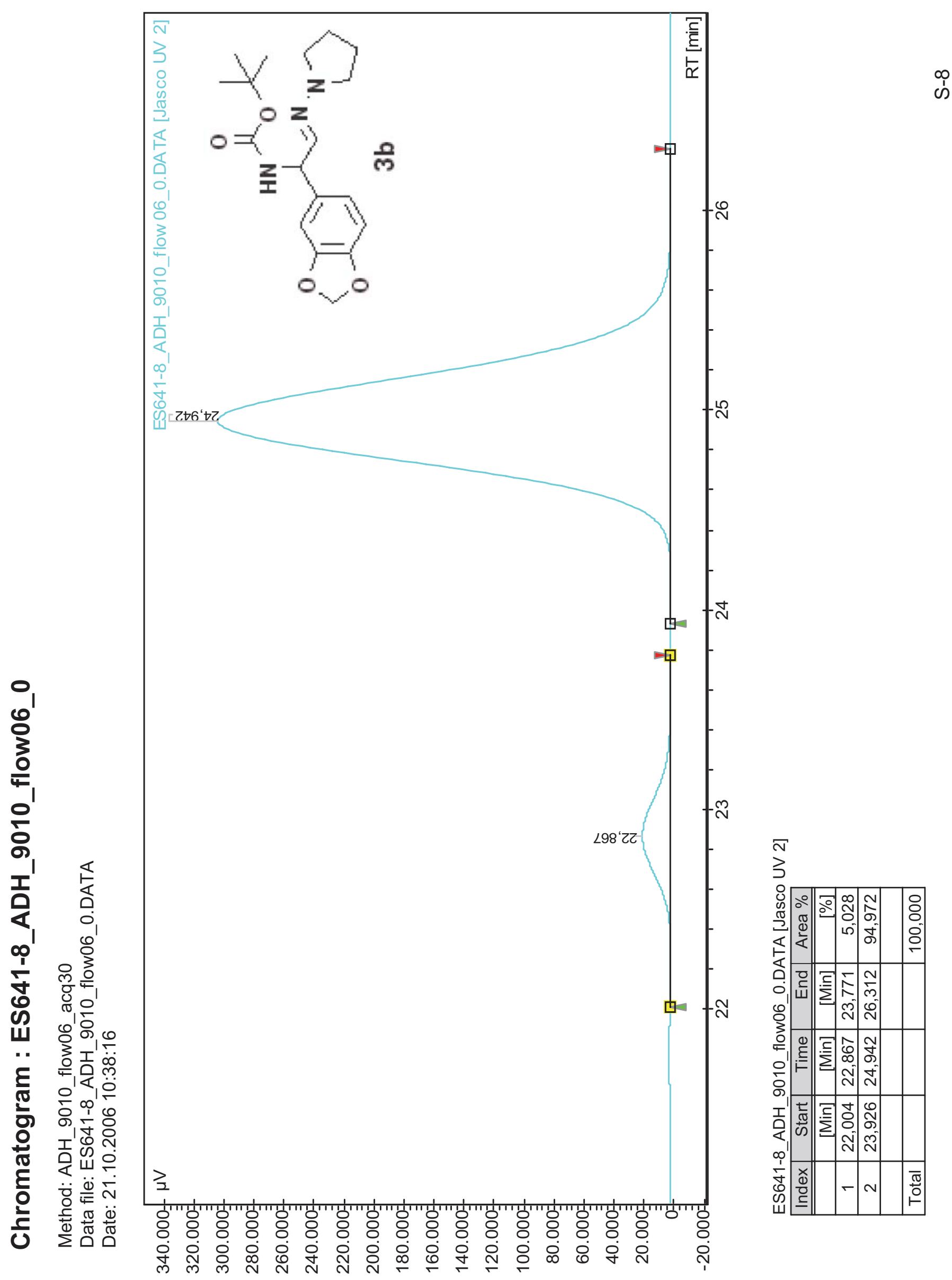




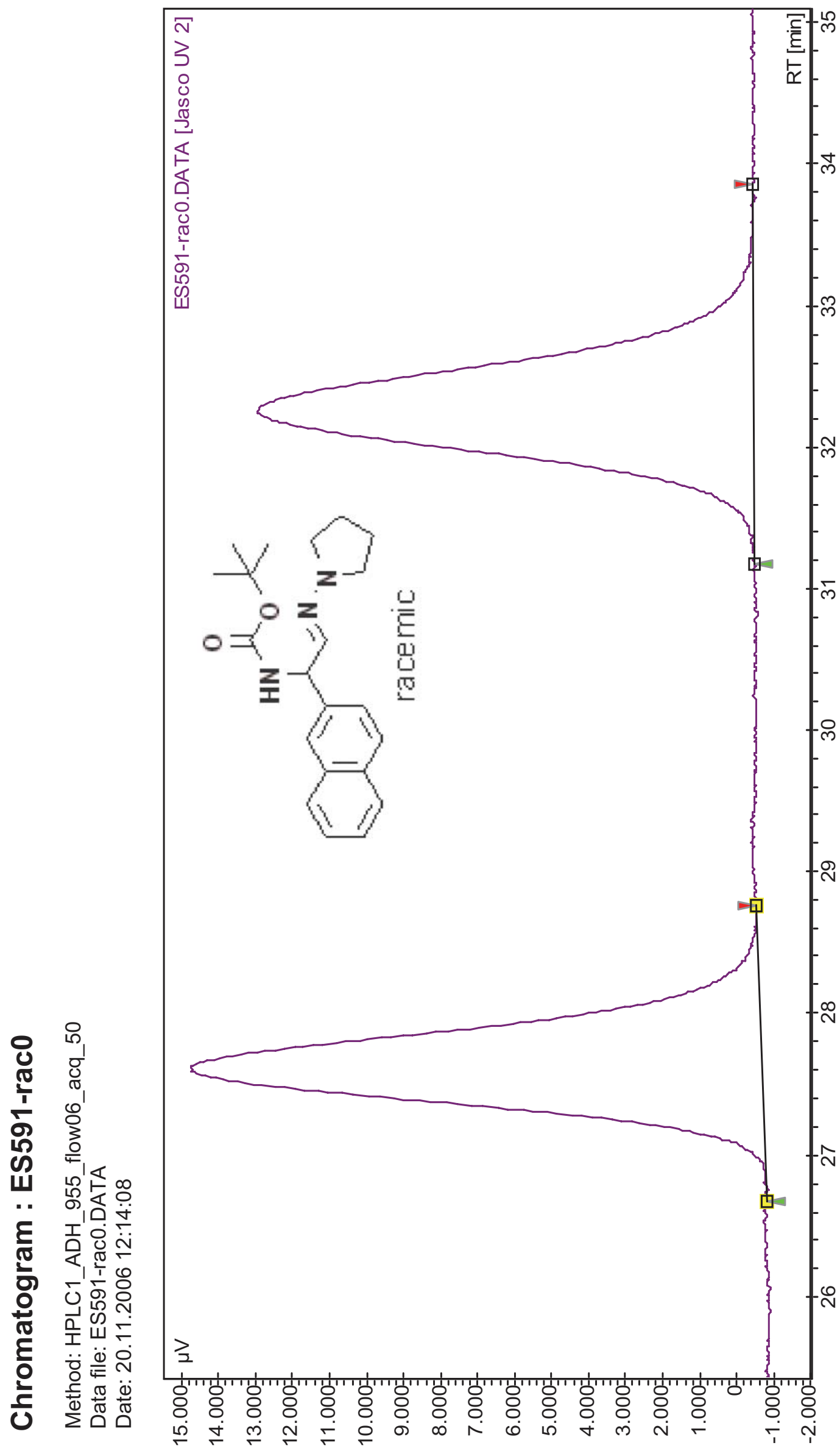

के

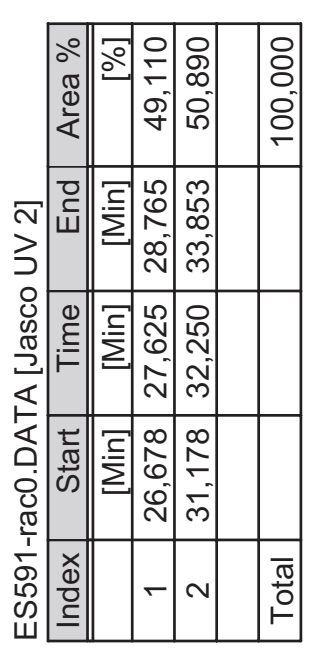




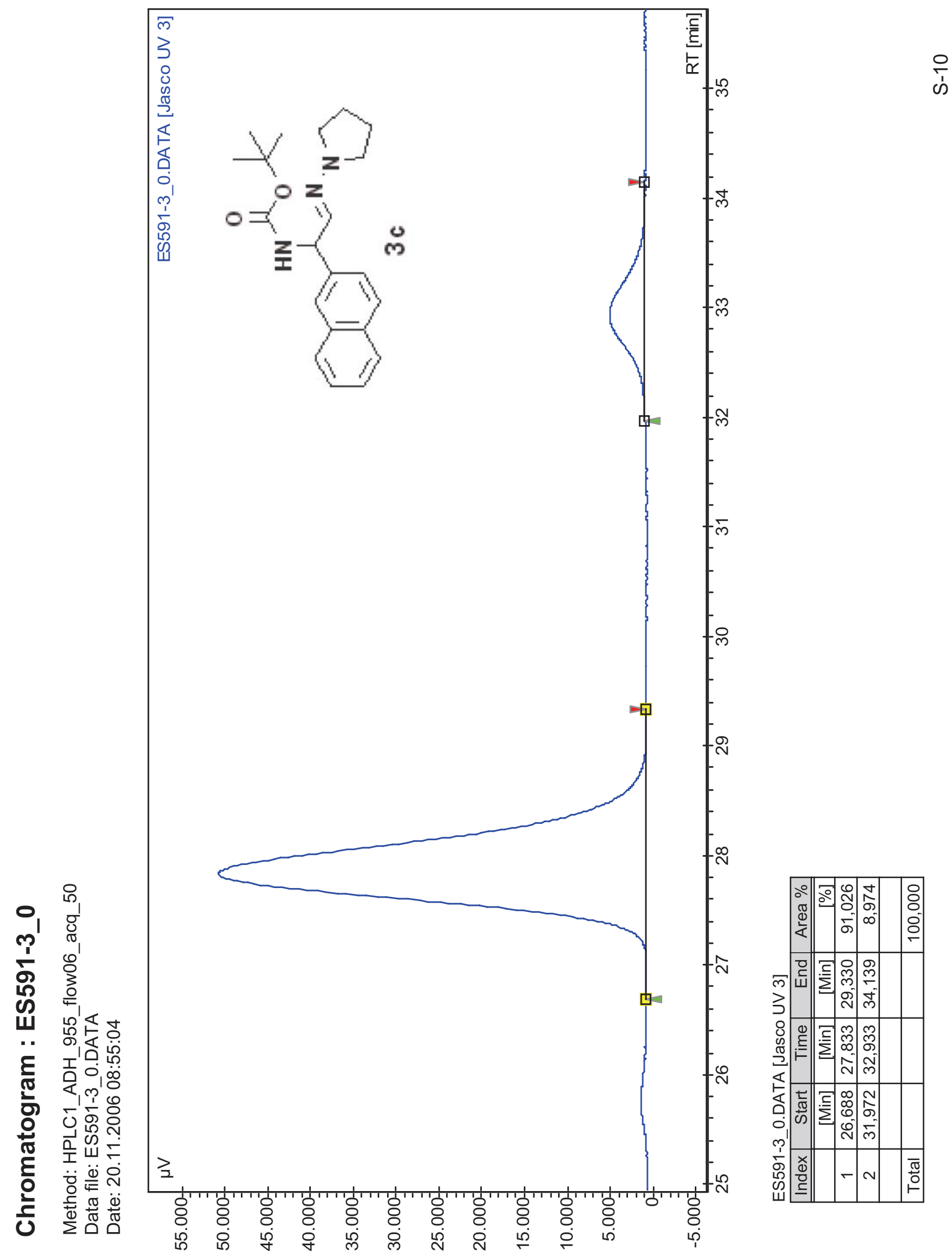




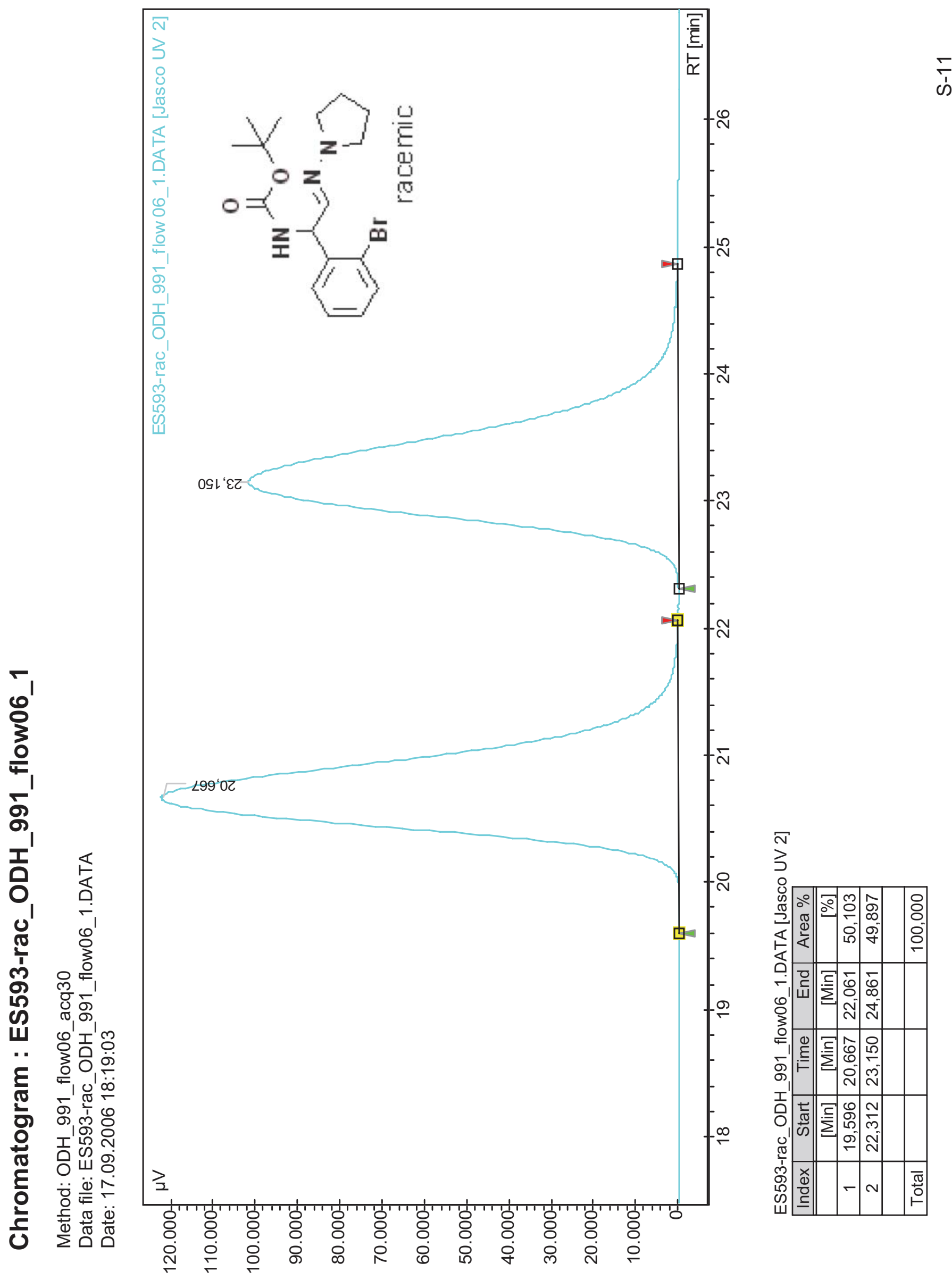




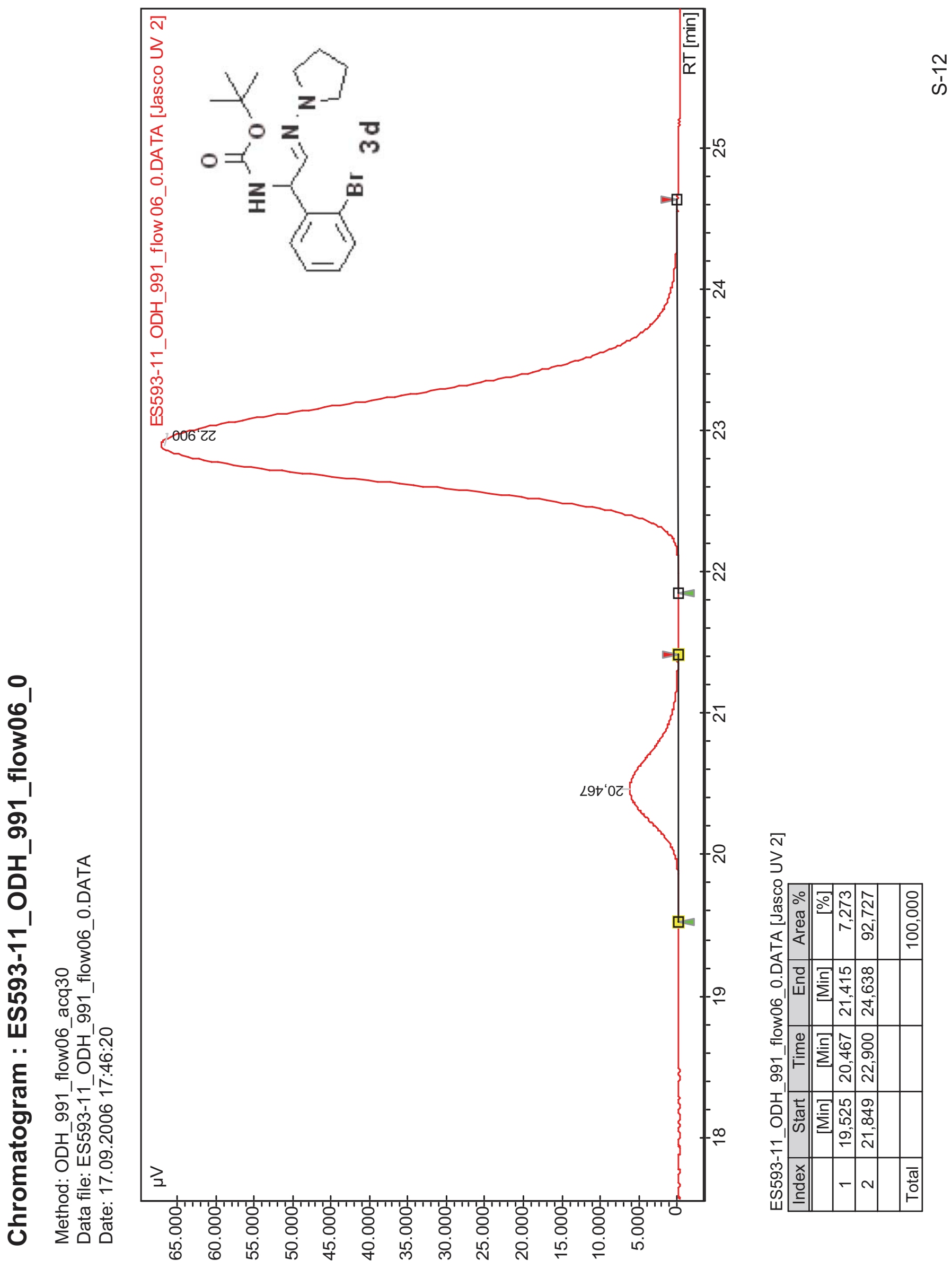




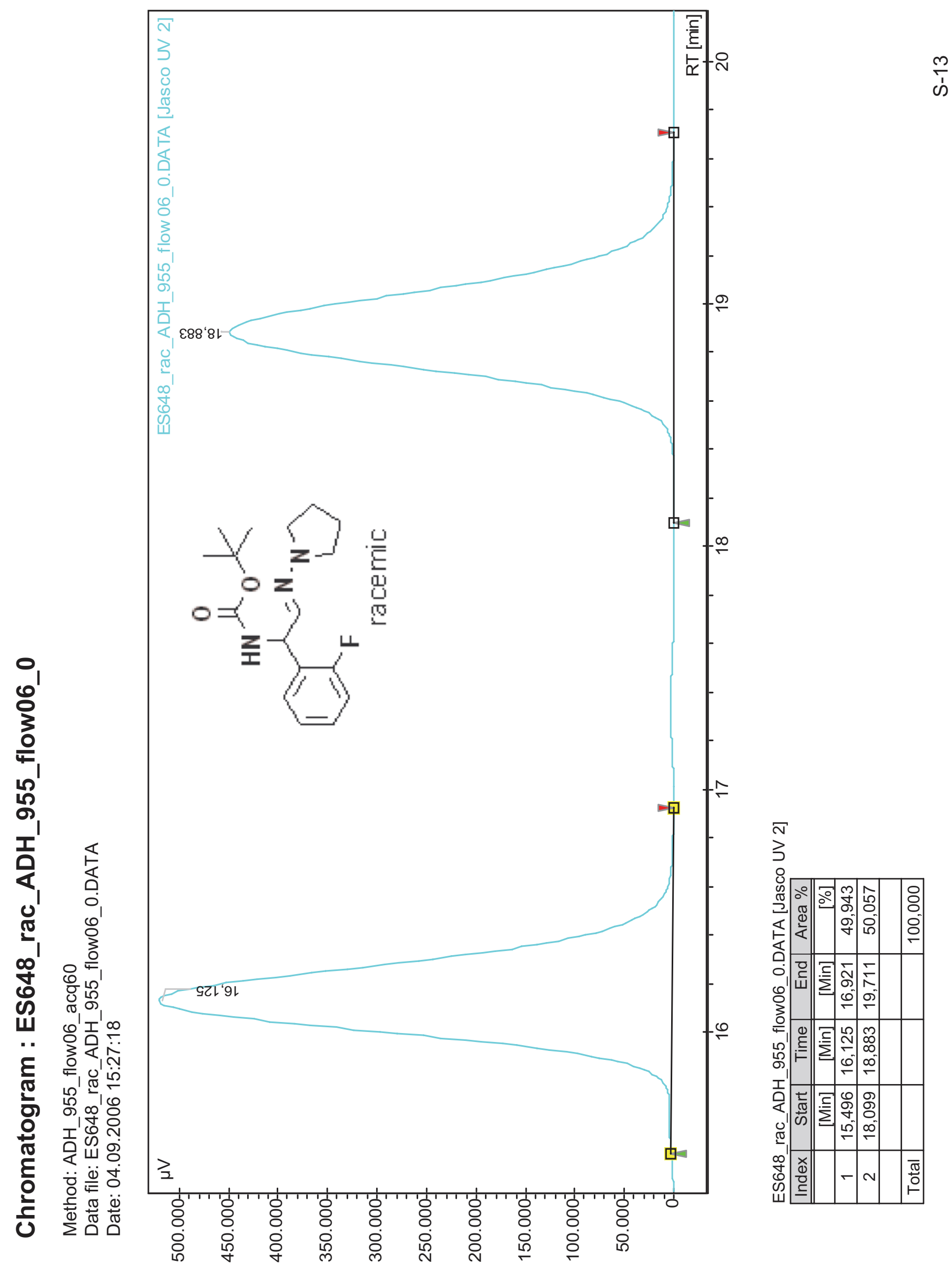




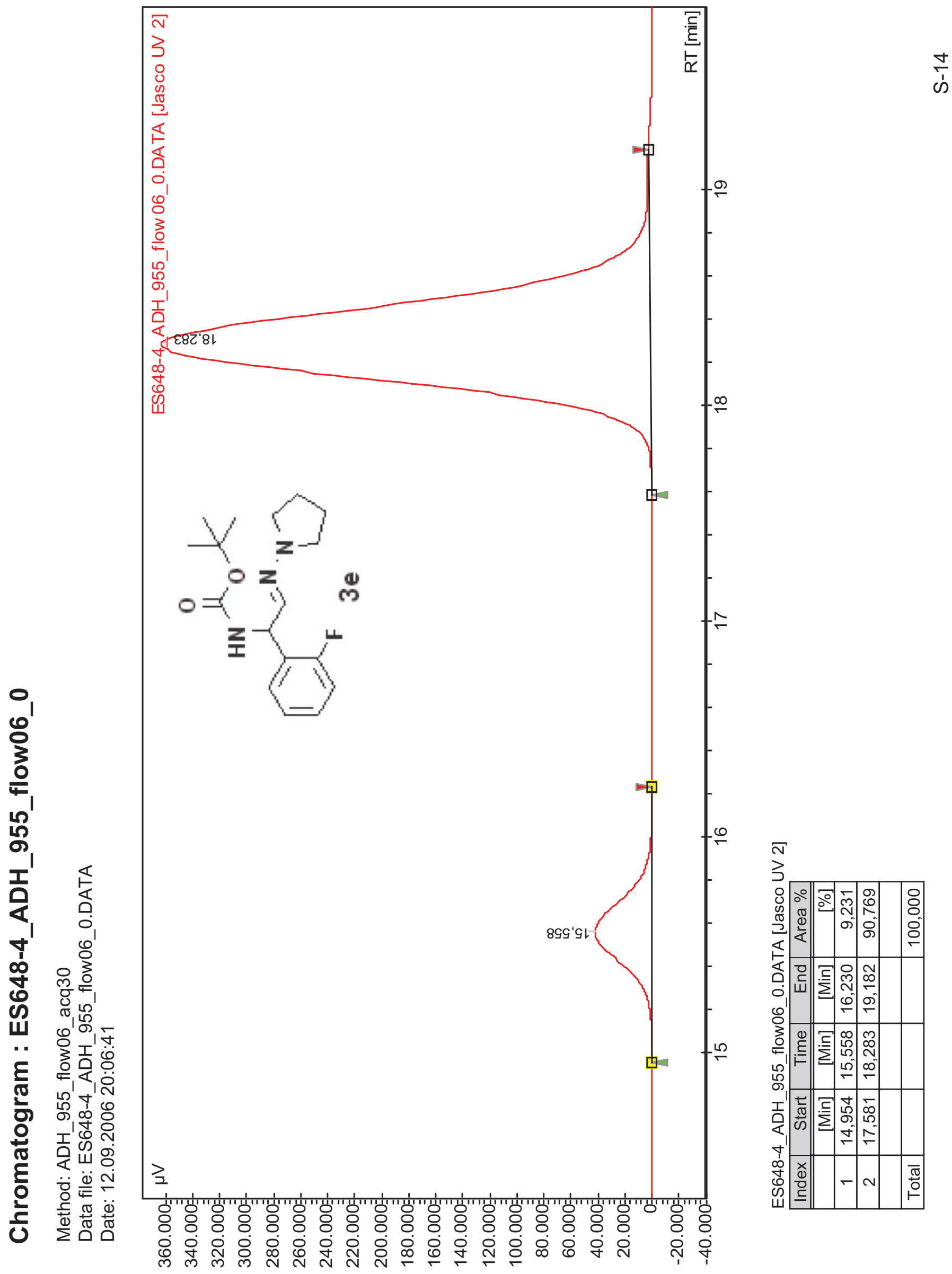




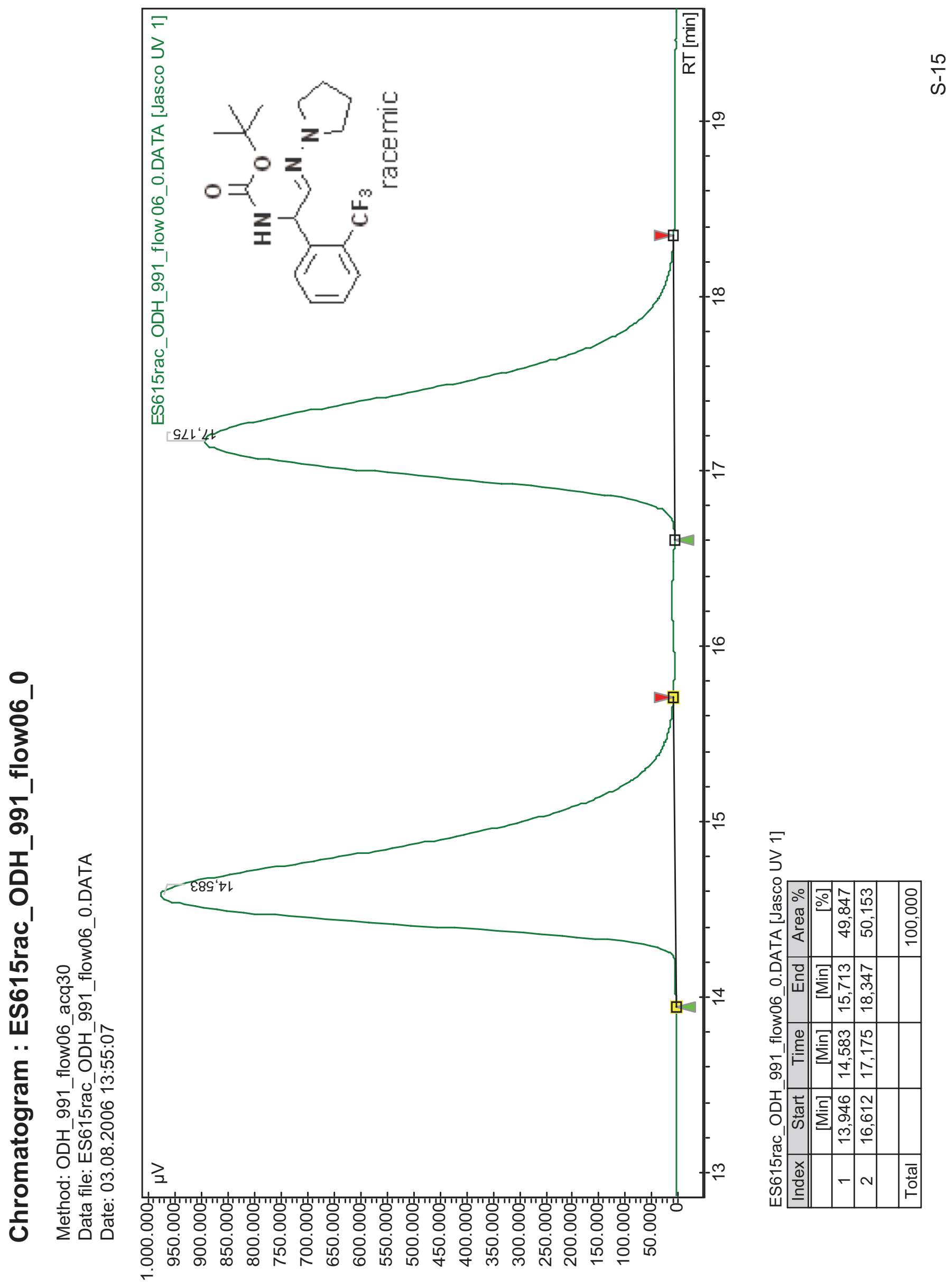




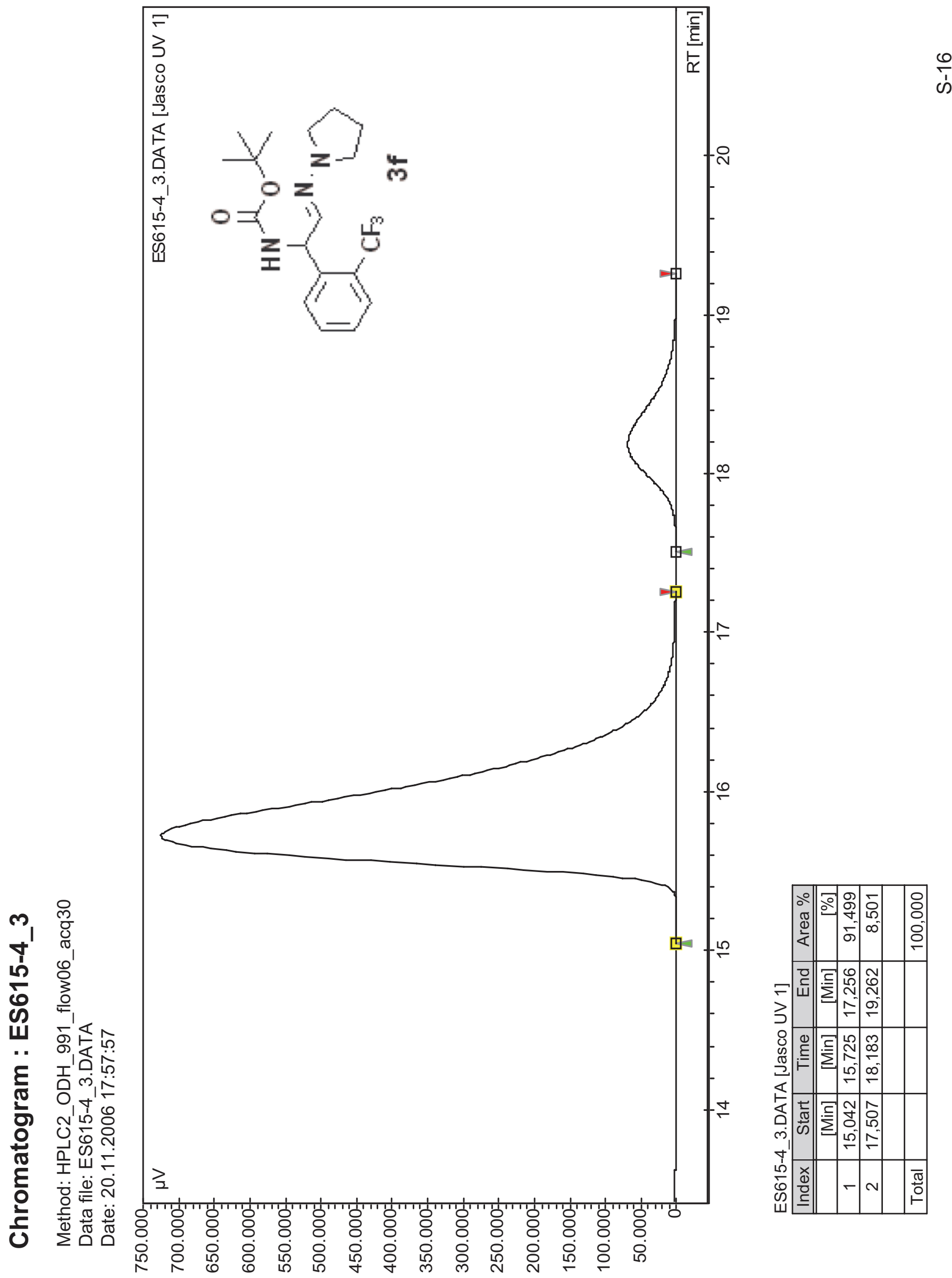




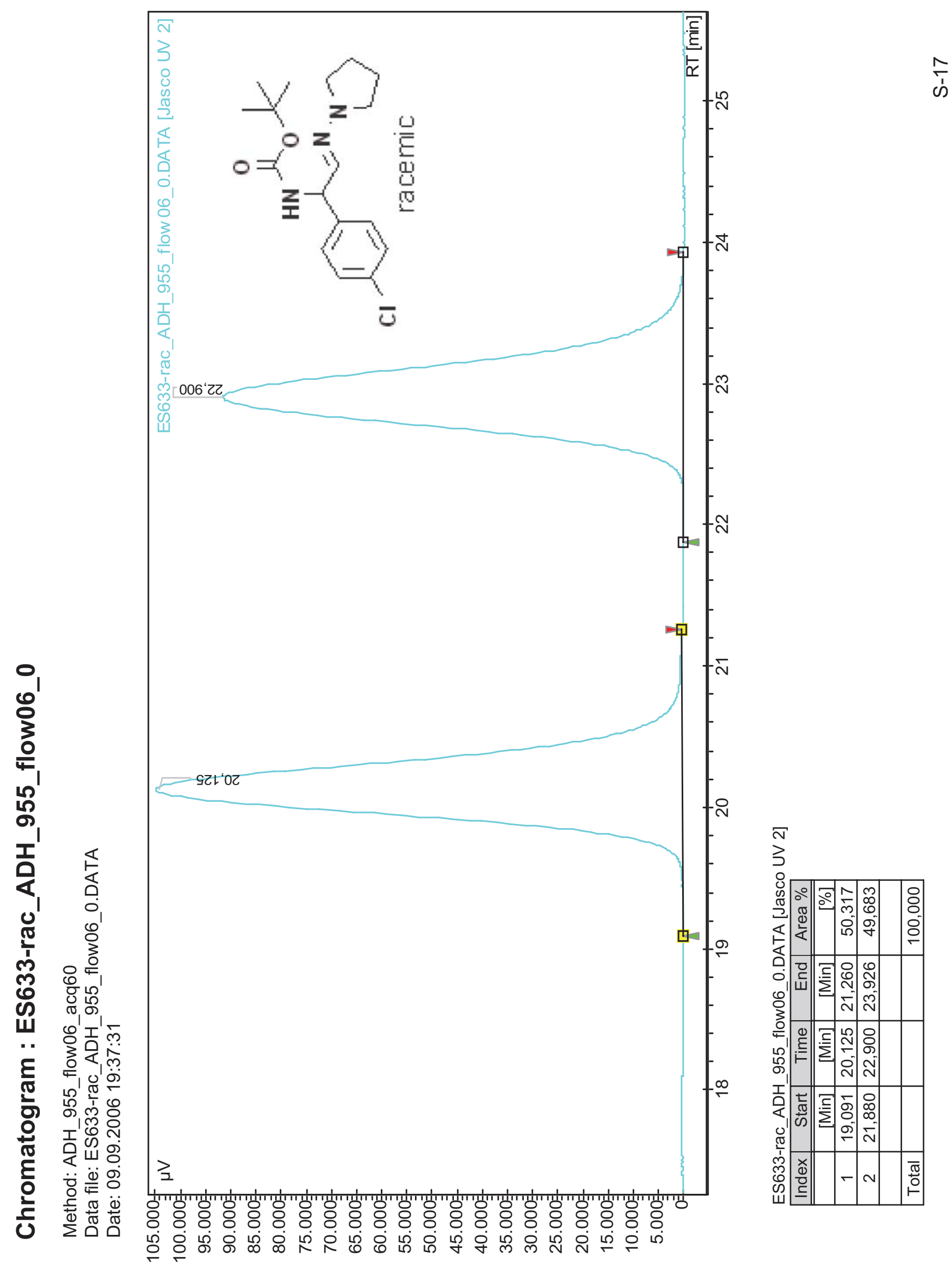




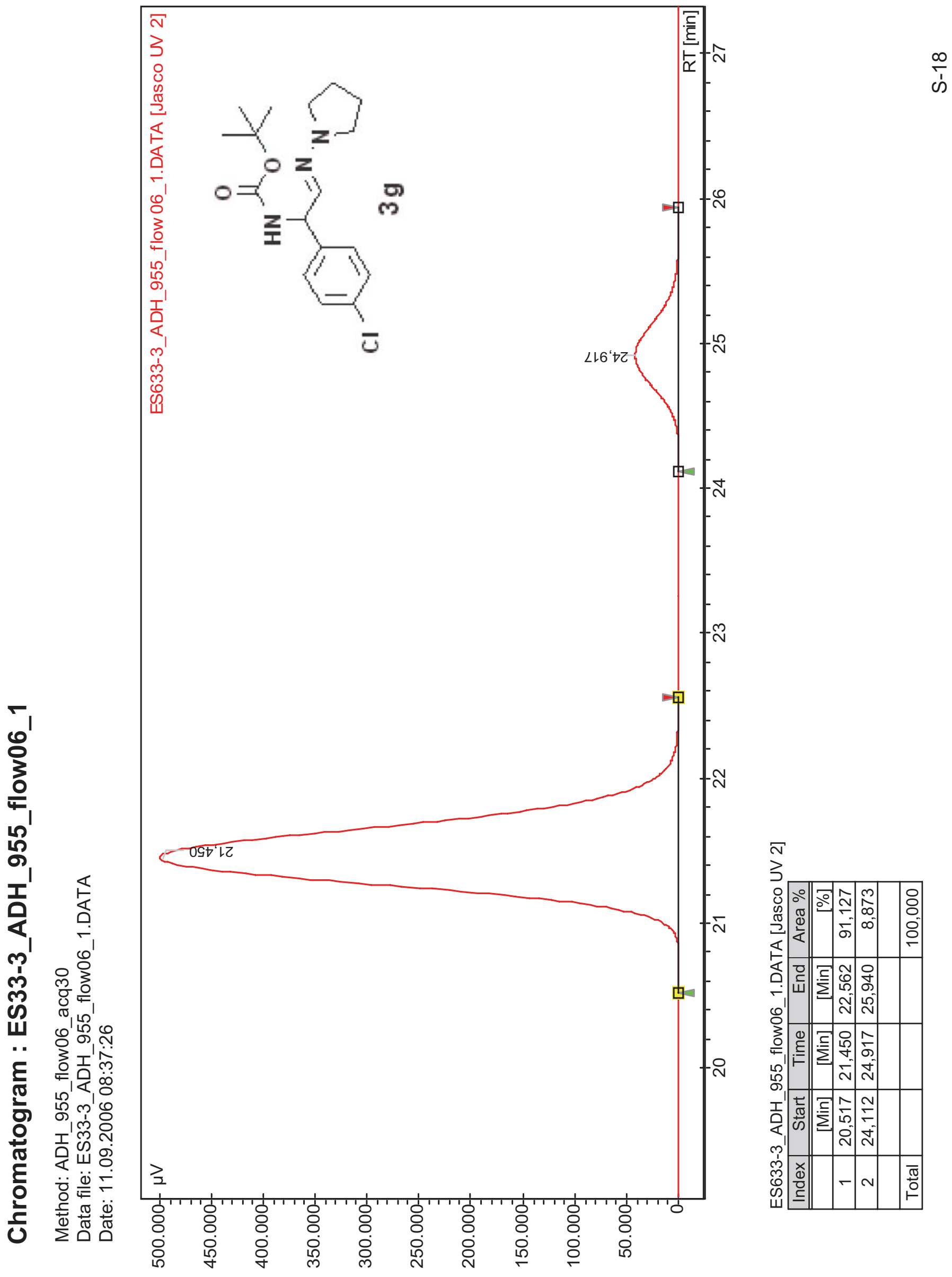




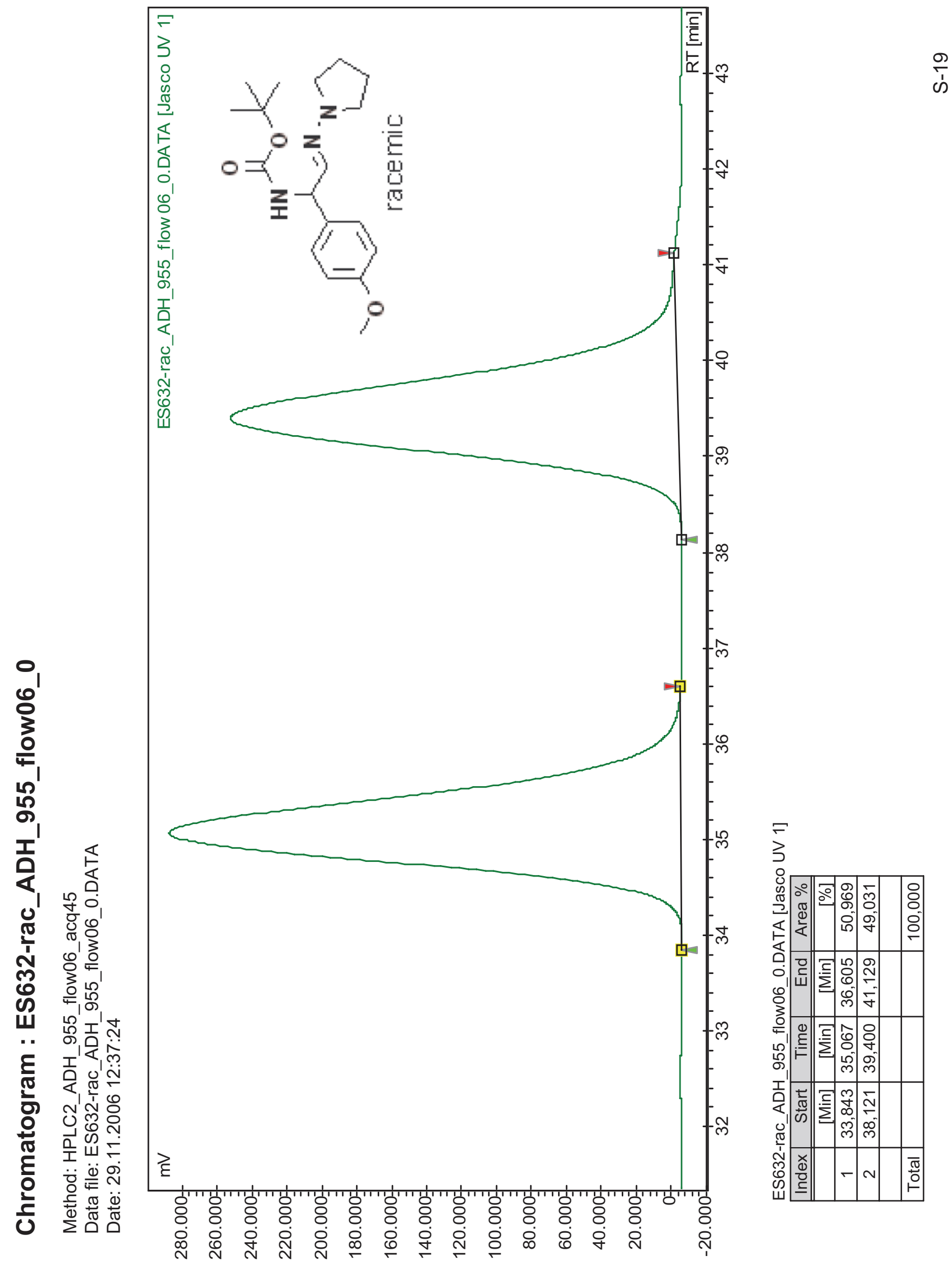




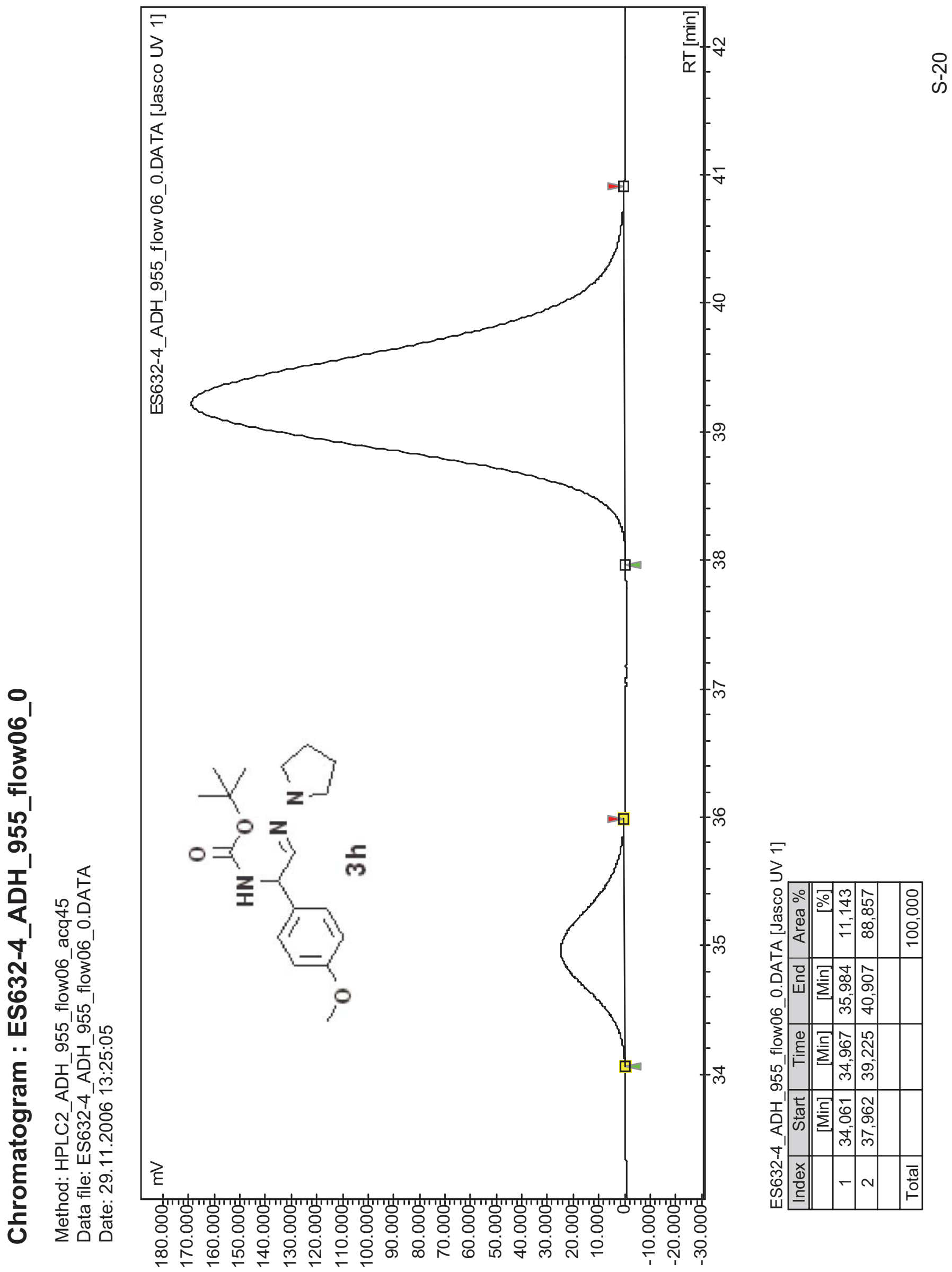




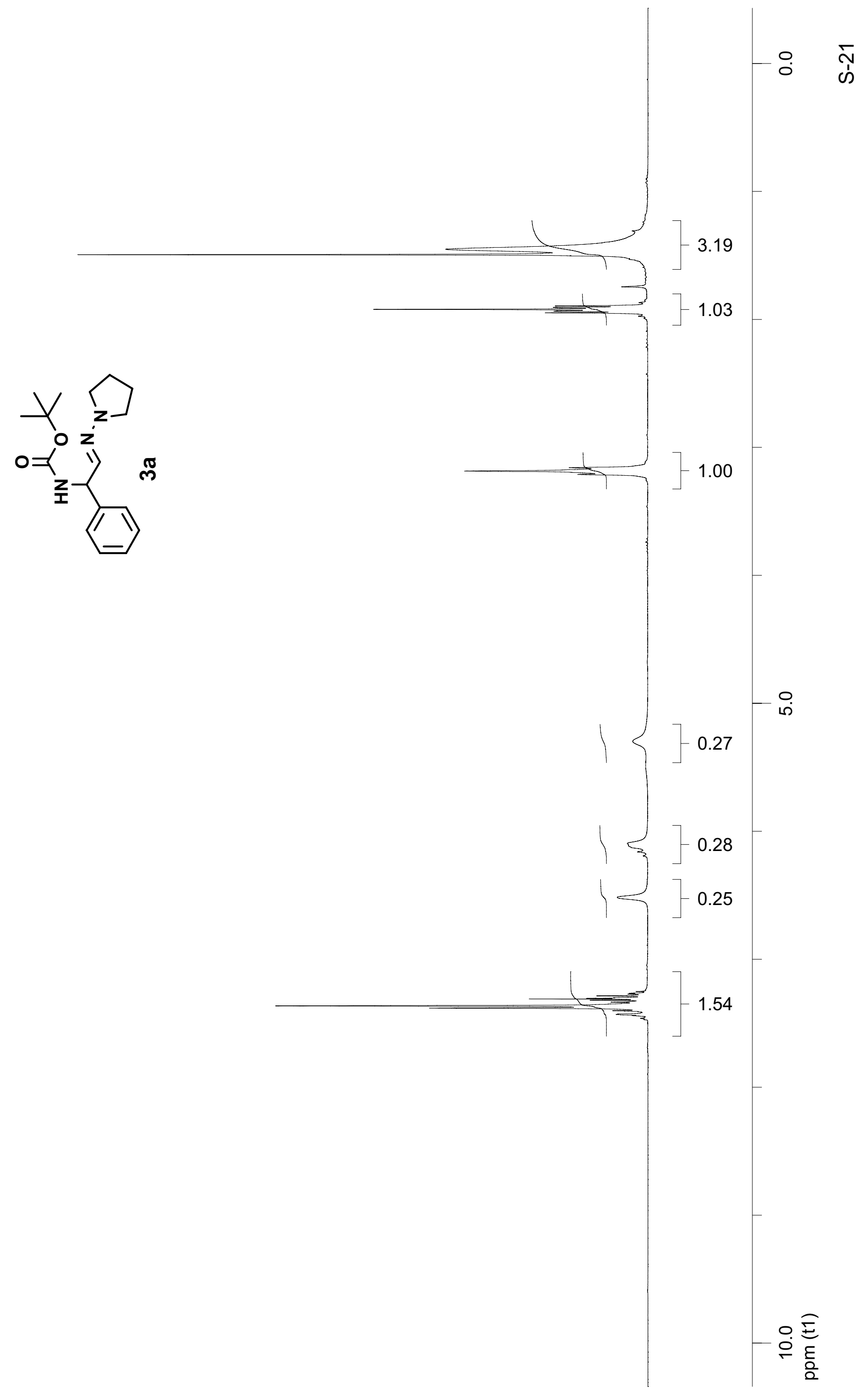




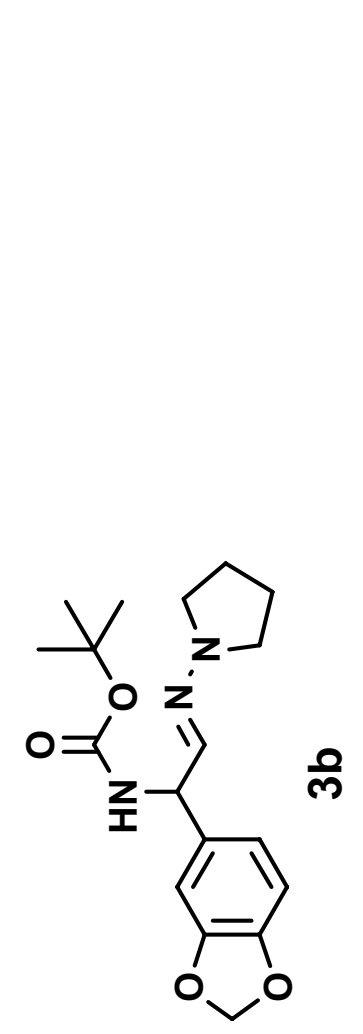




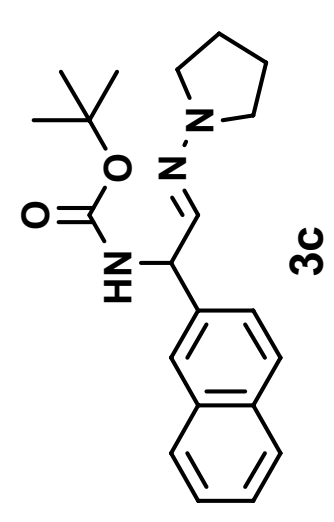




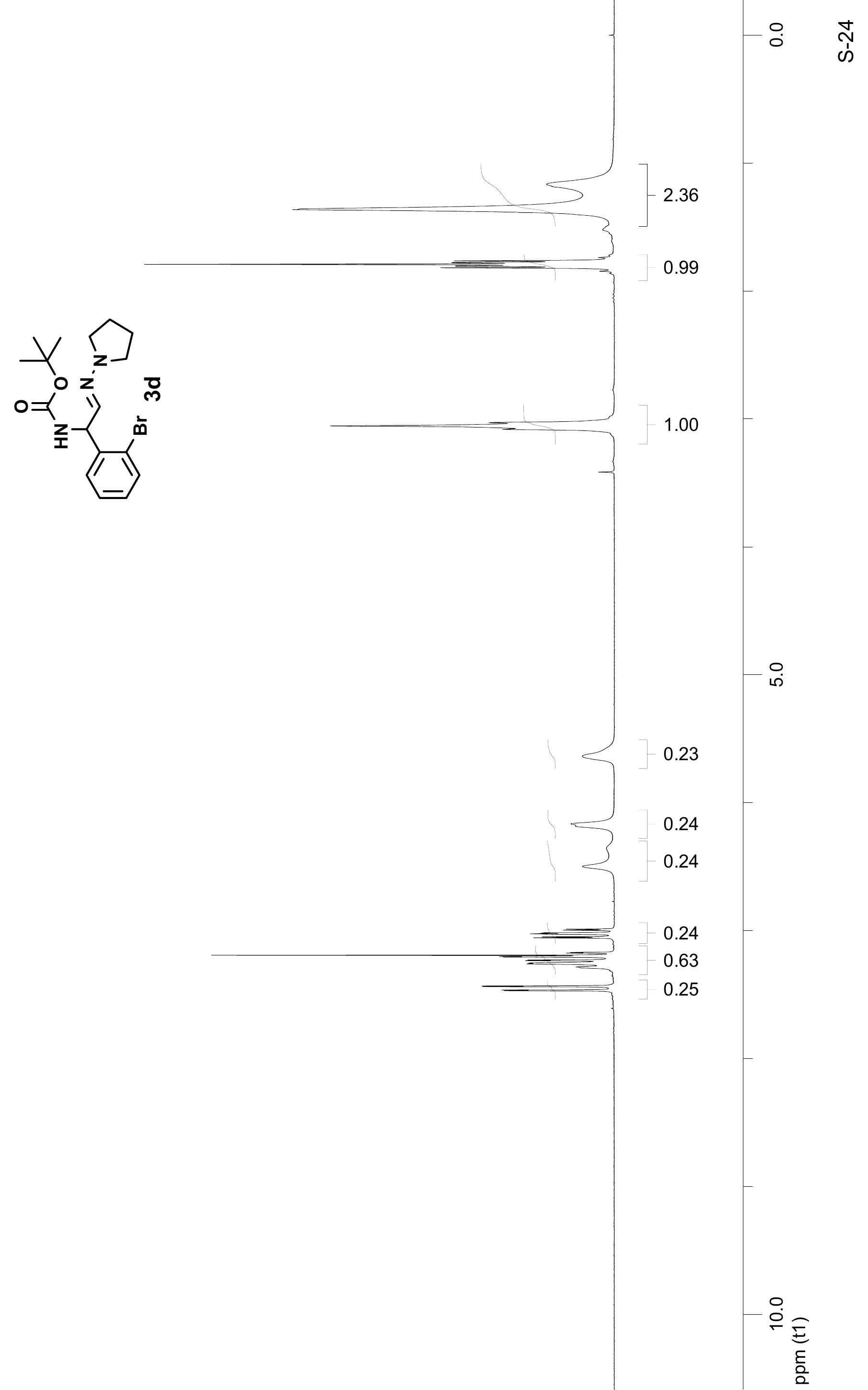




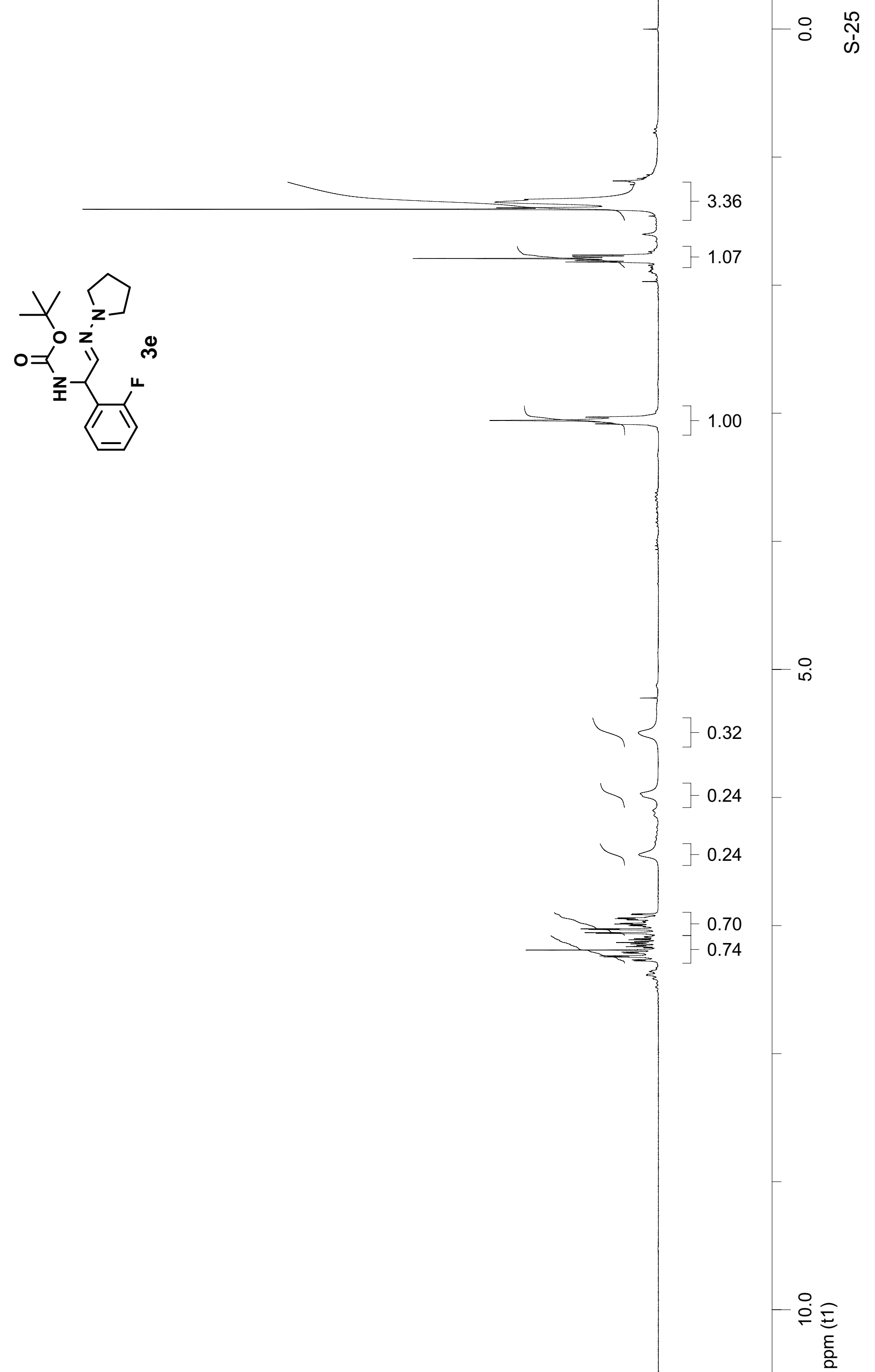




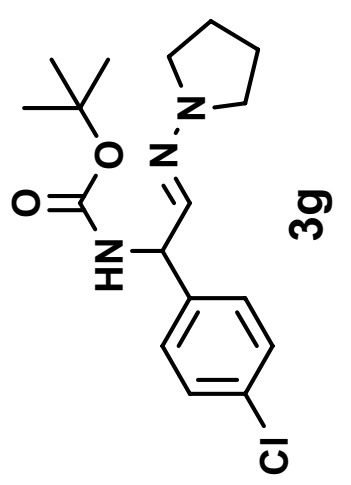



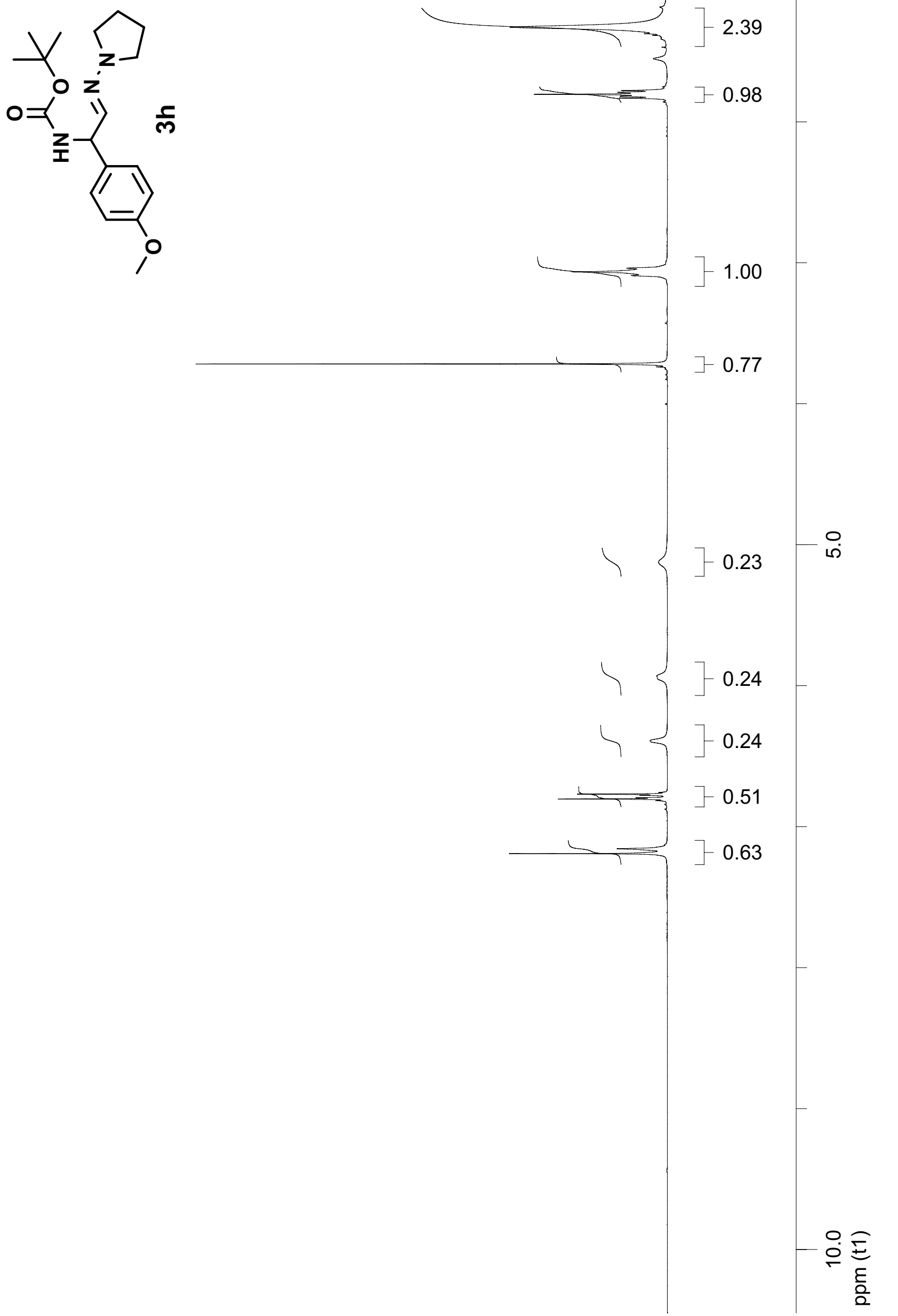
乙๕८

t.82

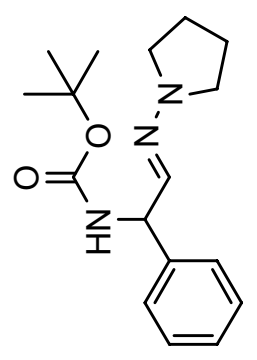

E'LS

$\nabla^{\circ} 6 L$

8'9टL

L $\angle Z L$

$\varepsilon ' \angle Z L$

$0.82 \mathrm{~L}$

9.8ZL

6.乙ยเ 
乙๕๕乙

t.82<smiles>[Z]C(=O)[C@H](C=C[C]1CCCC[C]1C(=O)OC(C)(C)C)c1ccc2c(c1)OCO2</smiles>

E'L

t $6 L$

乙.68

0.101

† $\angle O L$

$\varepsilon .80 \mathrm{~L}$

G.0ZL

6'乙ย

G'GEL

8.9tᄂ

$6 \angle \nabla L$

L.G

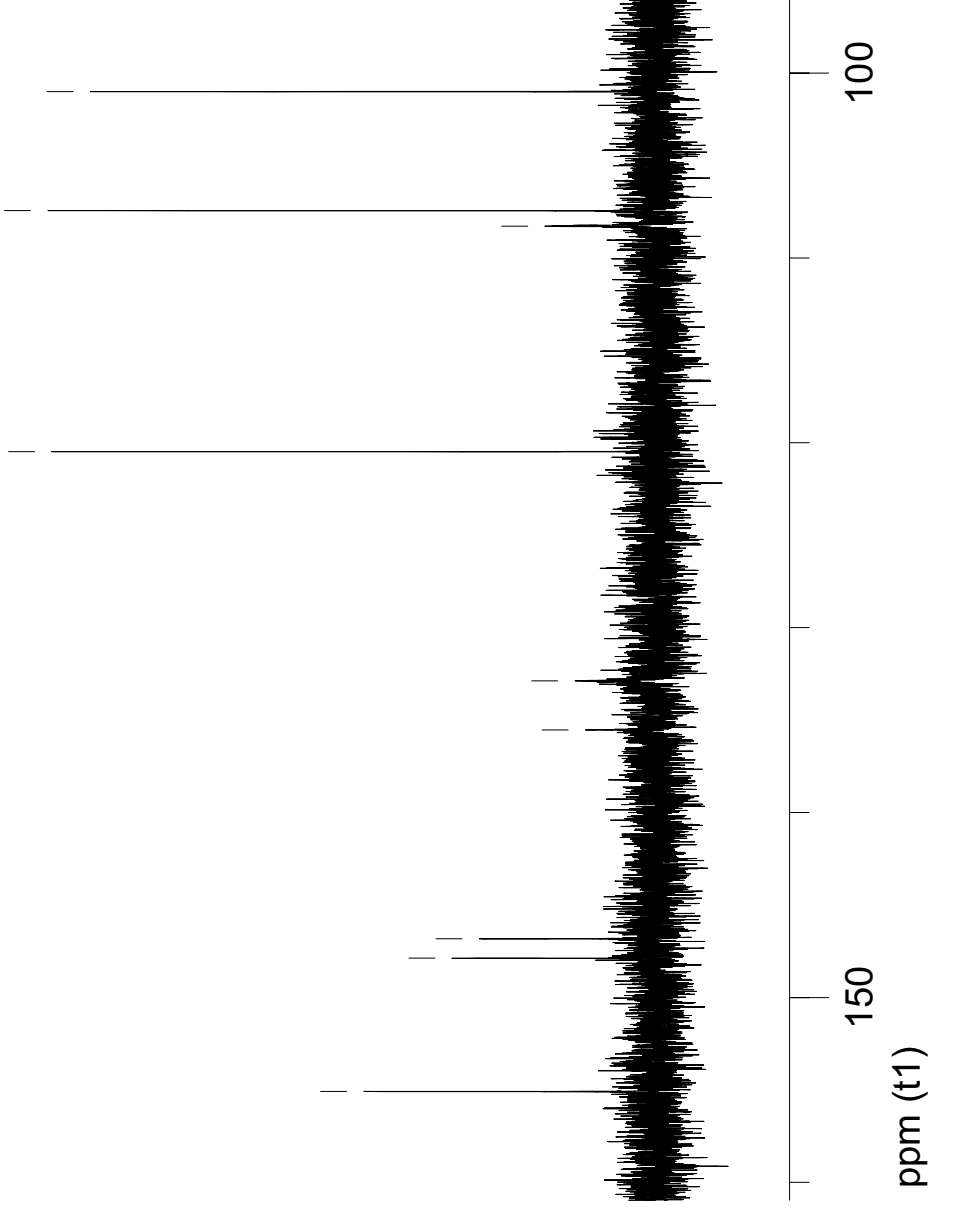


乙๕乙

t 82

E'LS

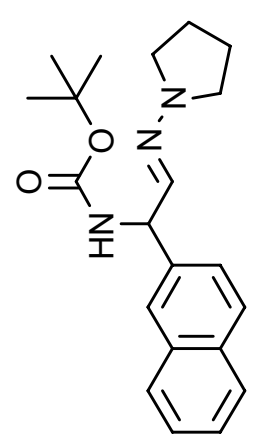

G'6L

† ๕นレ

†゙ォて

9.9ZL

乙.9ZL

$1.82 \mathrm{~L}$

6.8ZL

0 ㄴㄴ

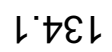

- 
$\varepsilon^{\circ} 8 乙$

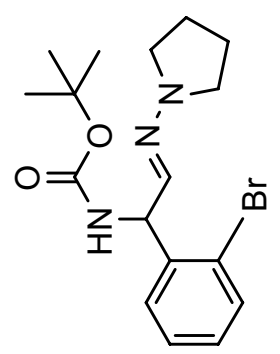

Eis

G'6L

8てZレ

6 LZL

9.8Z

L'OEL

$0^{\circ} \varepsilon \varepsilon\llcorner$ 
$\varepsilon^{\prime} 8 乙$

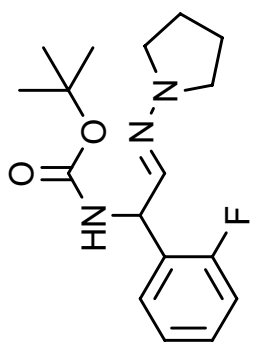

オ゙ォてレ

†゙ォレ

$0.82 \mathrm{~L}$

ㄷㄴ

8.8ZL

$\tau \cdot 8 \varepsilon$

$6^{\circ} \downarrow S$

E
$\frac{\varepsilon}{2}$ 
乙LเE乙

$\angle G \mathcal{E}^{\circ} 8 Z$

9ZZ IS

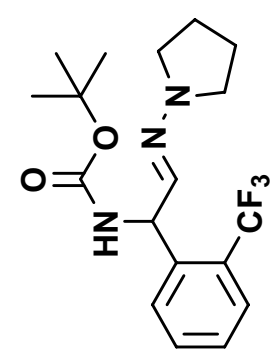

L8E $6 L$

乙レยててレ

0L9'9ZL

SL6 $9 Z$ L

†乙9 L乙L

6Lع'乙ع 
$\angle \triangleright 乙 \varepsilon 乙$

$86 \varepsilon^{\circ} 82$

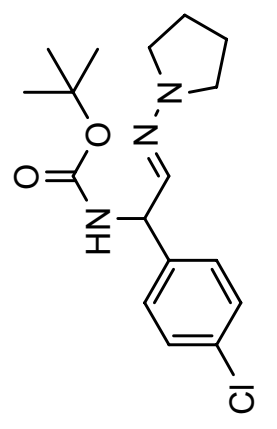

LGZLG

$929^{\circ} 62$

0เ乙'8ZL

6t7'8ZL

\&†L'8ZL

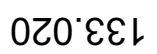


乙๕८

$t^{\circ} 82$

E'L

$\varepsilon$ ¿ 9

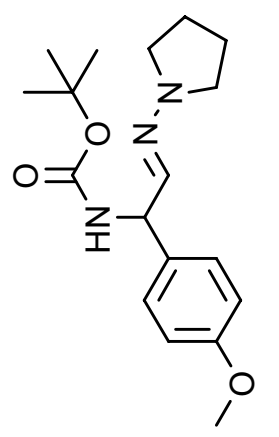

ले
ஸे

유

음

0๋レレ

ع.8ZL

G'Eع

เ'GS

6.891

E

ํํㅇ 


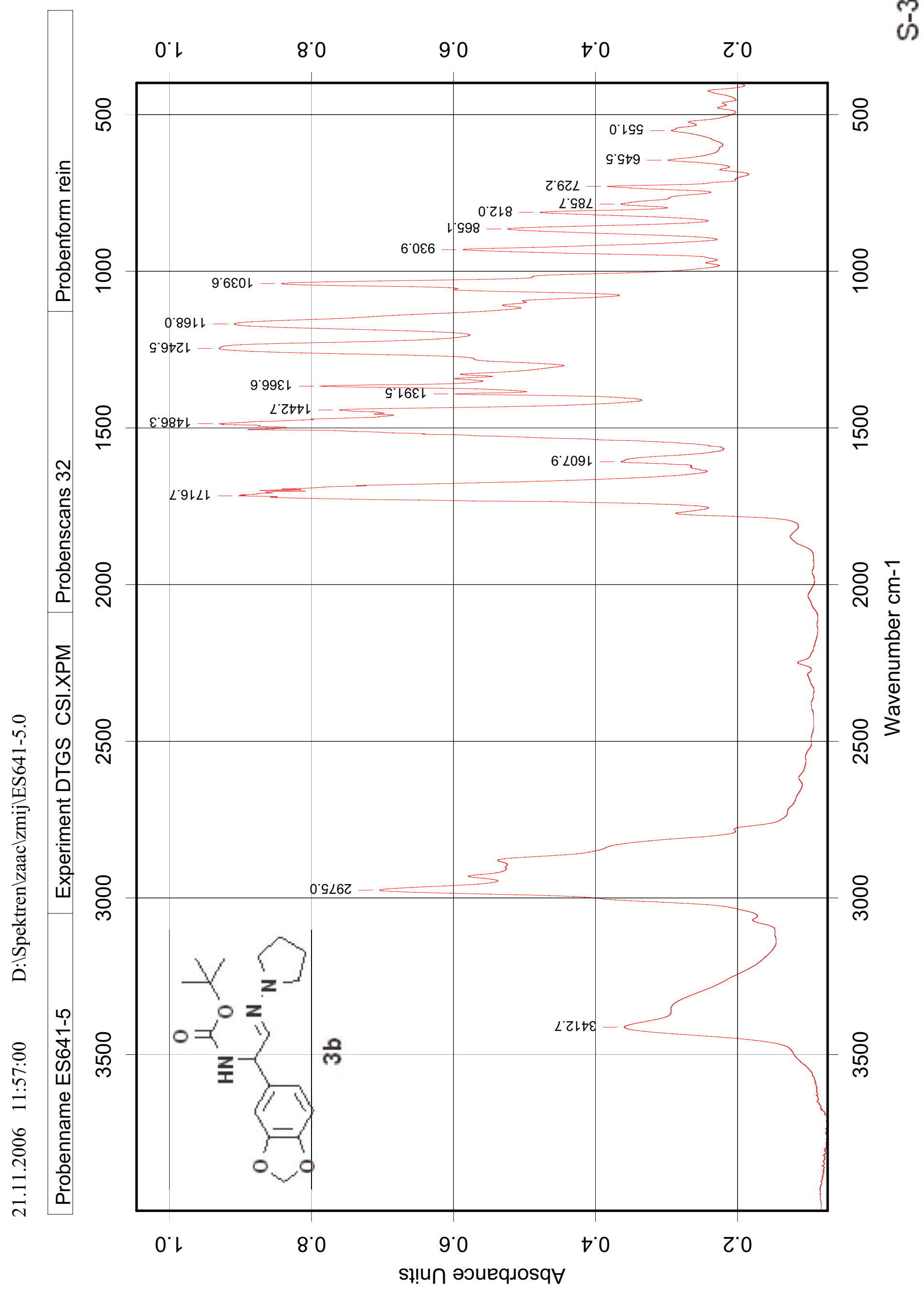




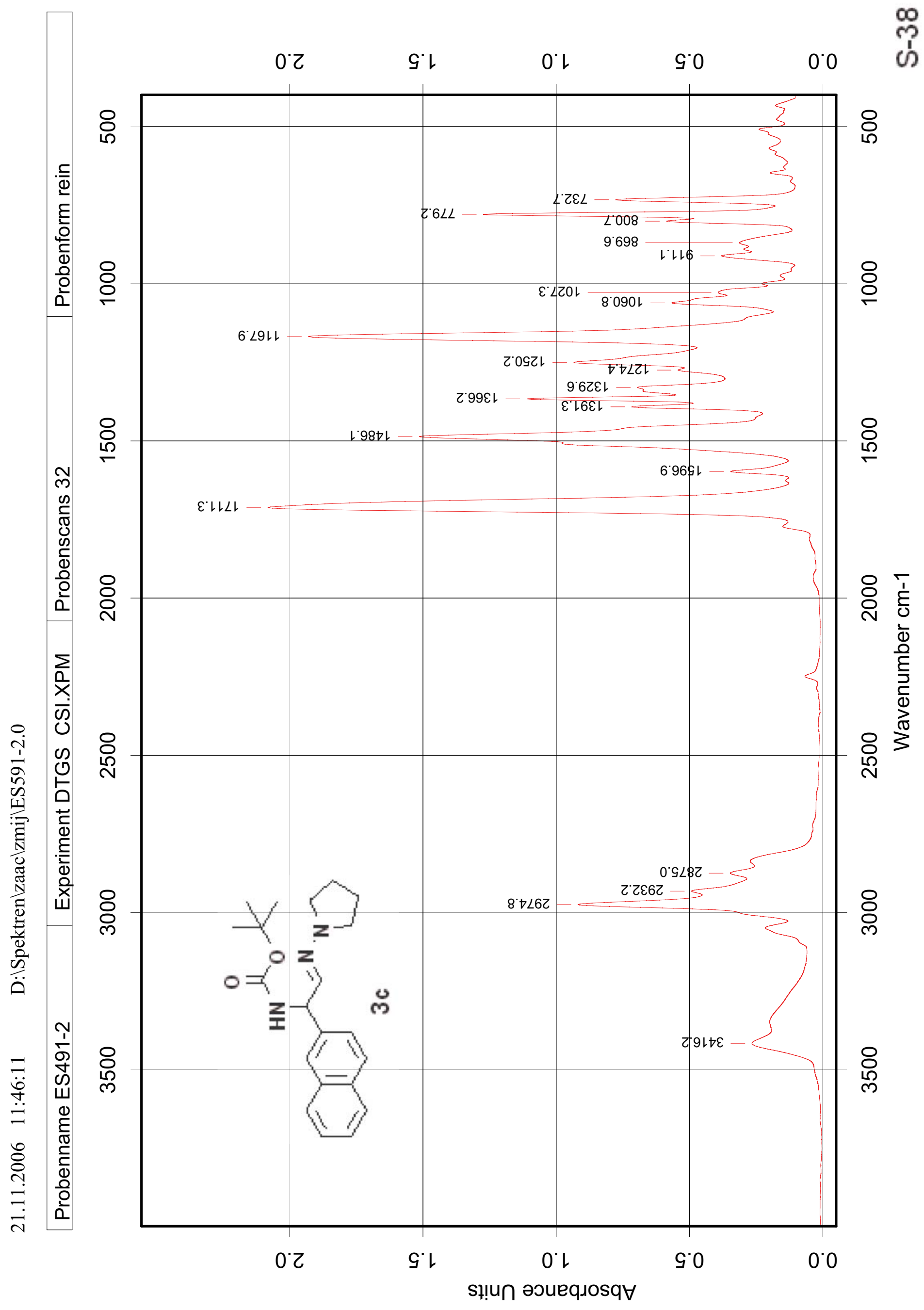




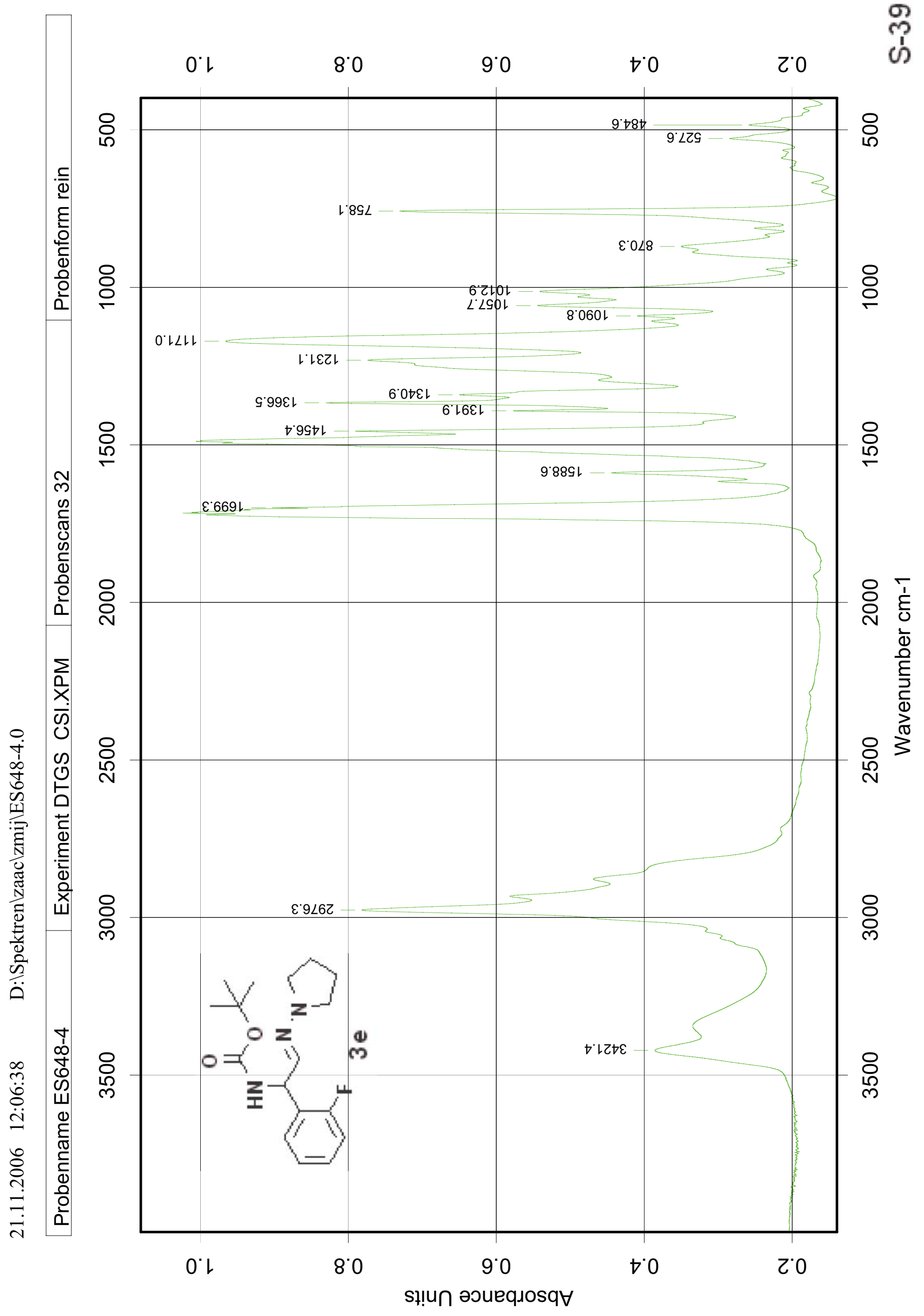




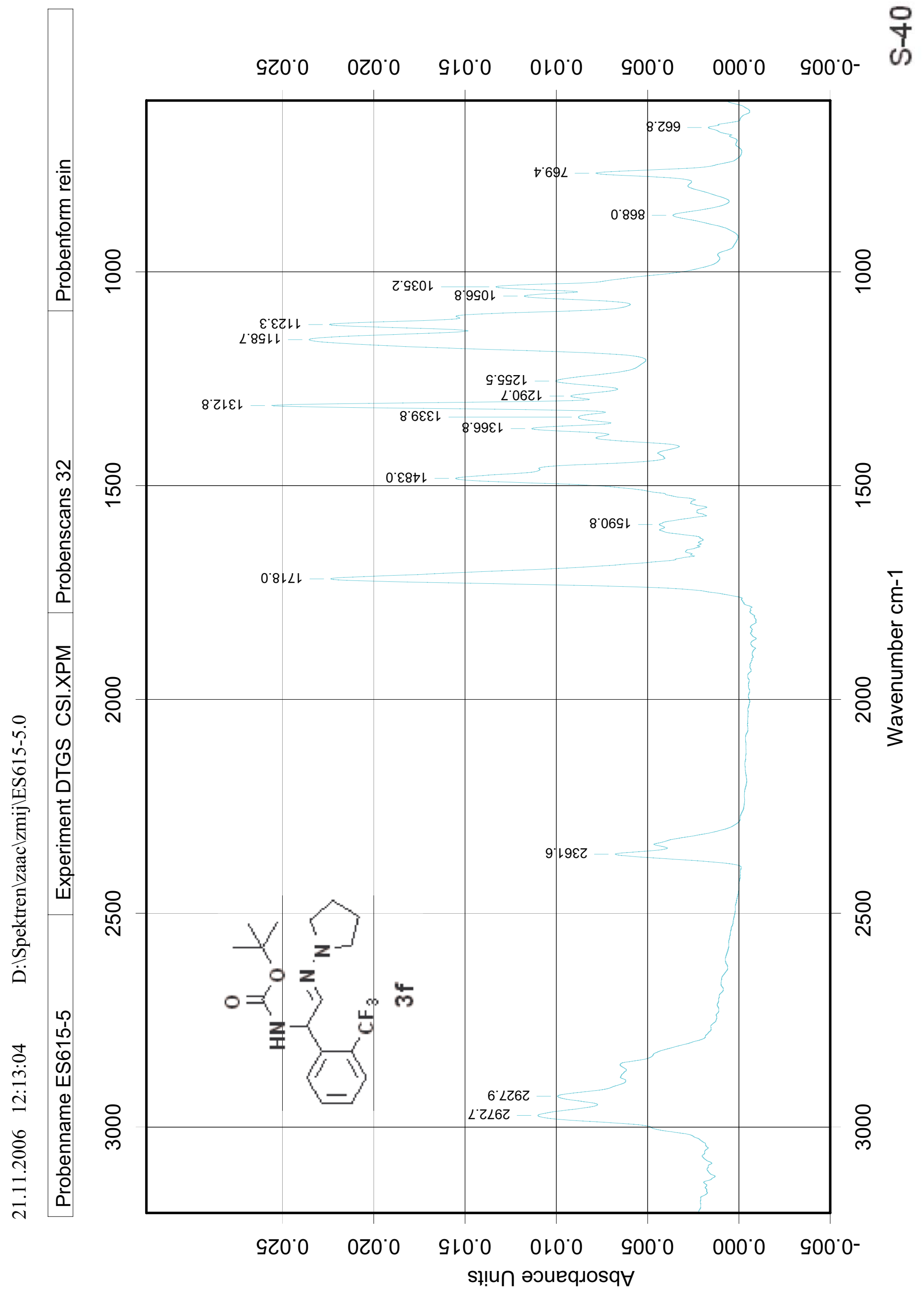




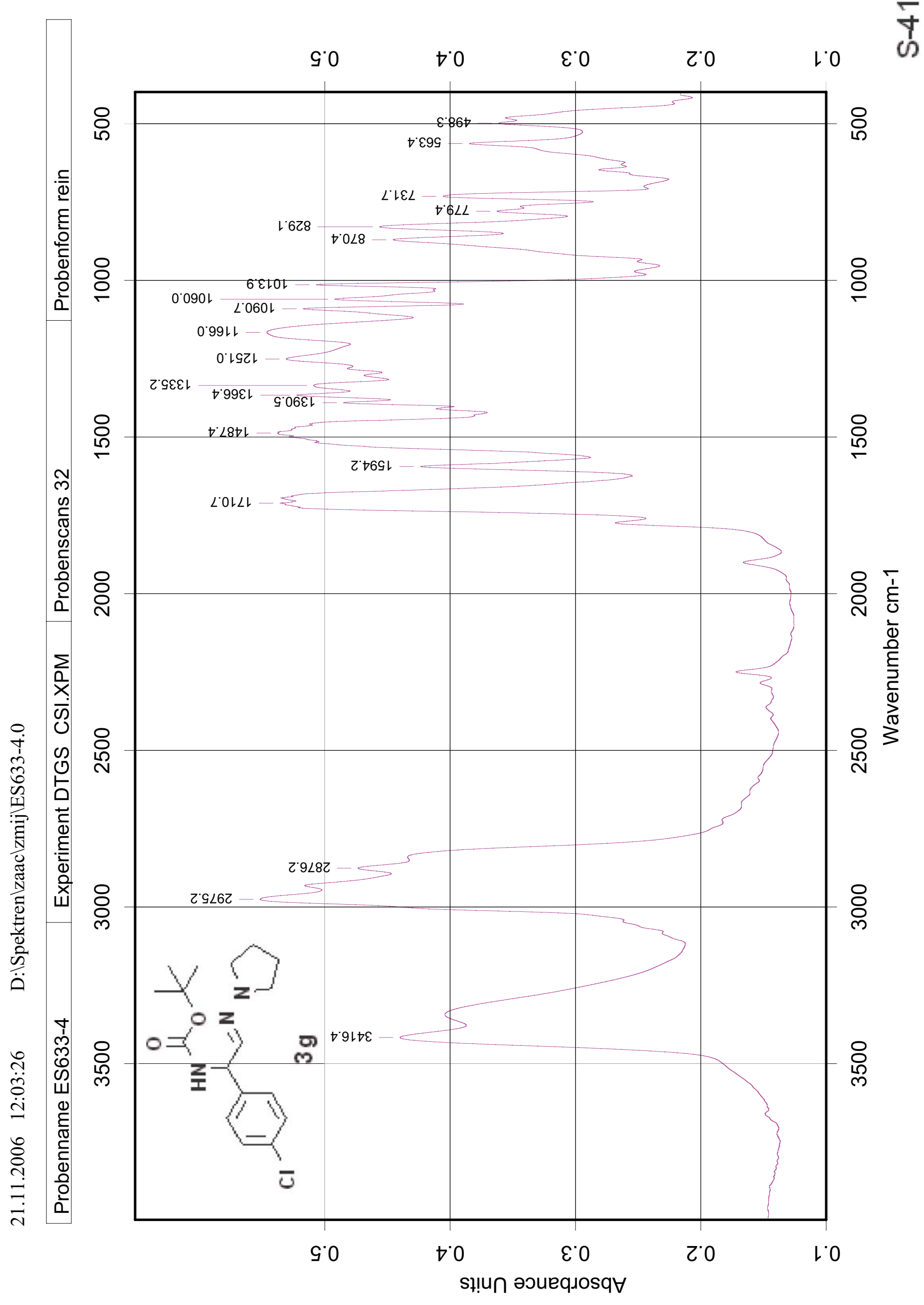




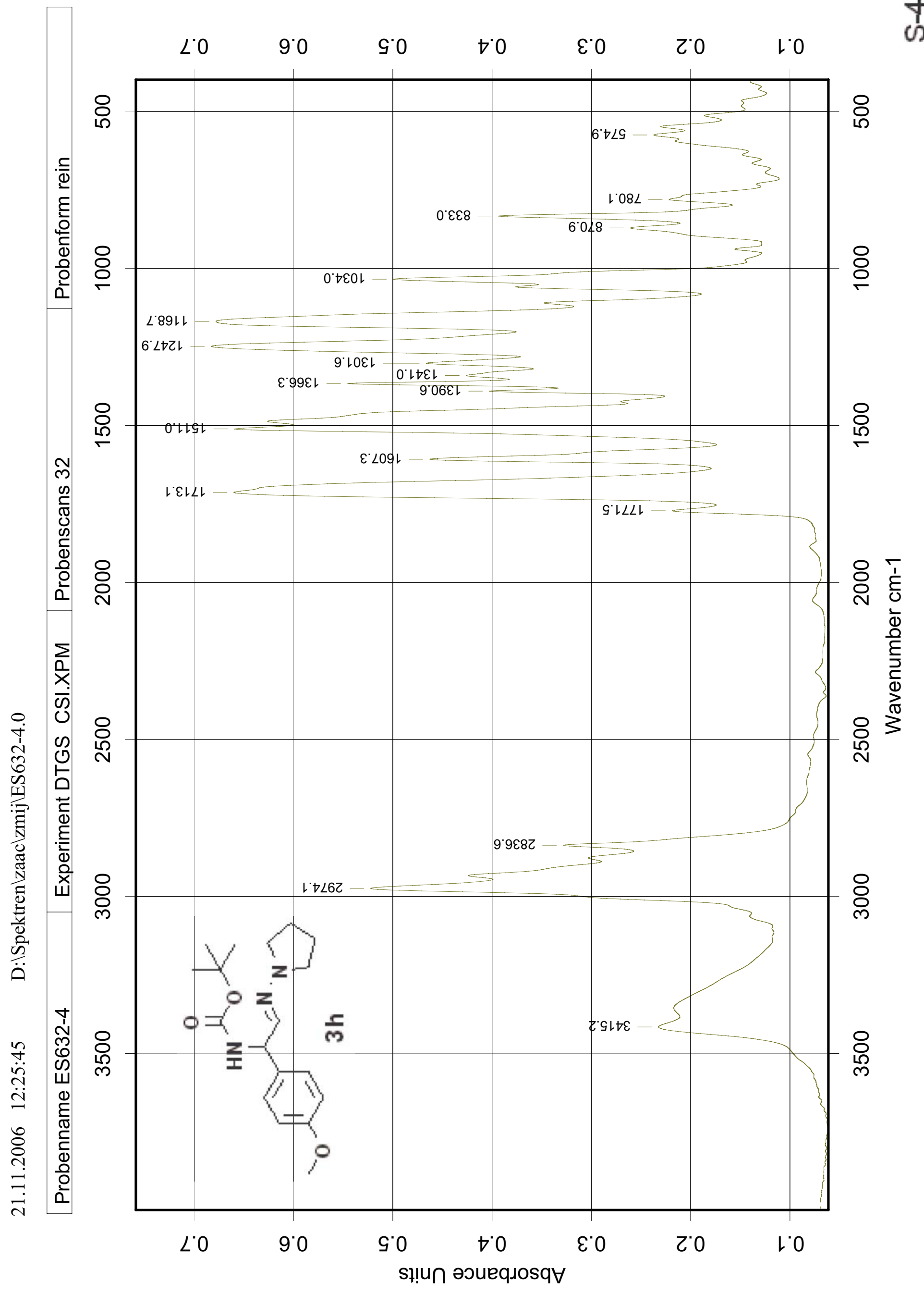

